\title{
ON THE DIRICHLET PROBLEM OF MIXED TYPE FOR LOWER HYBRID WAVES IN AXISYMMETRIC COLD PLASMAS
}

\author{
DANIELA LUPO, DARIO D. MONTICELLI, AND KEVIN R. PAYNE
}

\begin{abstract}
For a class of linear second order partial differential equations of mixed elliptic-hyperbolic type, which includes a well known model for analyzing possible heating in axisymmetric cold plasmas, we give results on weak well-posedness of the Dirichlet problem and show that such solutions are characterized by a variational principle. The weak solutions are shown to be saddle points of natural functionals suggested by the divergence form of the PDEs. Moreover, the natural domains of the functionals are the weighted Sobolev spaces to which the solutions belong. In addition, all critical levels will be characterized in terms of global extrema of the functionals restricted to suitable infinite dimensional linear subspaces. These subspaces are defined in terms of a robust spectral theory with weights which is associated to the linear operator and is developed herein. Similar characterizations for the weighted eigenvalue problem and nonlinear variants will also be given. Finally, topological methods are employed to obtain existence results for nonlinear problems including perturbations in the gradient which are then applied to the well-posedness of the linear problem with lower order terms.
\end{abstract}

\section{Introduction}

The Dirichlet problem of mixed elliptic-hyperbolic type

$$
\begin{cases}\left(\left(x-y^{2}\right) u_{x}\right)_{x}+u_{y y}=f & \text { in } \Omega, \\ u=\phi & \text { on } \partial \Omega\end{cases}
$$

where $f$ and $\phi$ are given and $\Omega \subset \mathbb{R}^{2}$ has nontrivial intersection with the sets $\left\{ \pm\left(x-y^{2}\right)>0\right\}$ has been proposed by Weitzner [28. (see also [27]) as a natural model for studying the possibility of heating in cold plasmas. In this model, one studies linear 3-D propagation of applied high frequency waves in a plasma, thought of as a dielectric medium populated by electrons and one or more species of ions, which carry electric charge and current, whose mass and momentum are conserved and where Maxwell's equations involving the dielectric tensor close the system. Under axisymmetry, the cold plasma model can be written in cylindrical coordinates $(r, \theta, z)$ as a coupled system of second order PDEs for the angular components $\left(E_{\theta}, B_{\theta}\right)=(v, u)$ of the electric and magnetic fields (which then determine the other field components through a first order system of PDEs). The equation for $E_{\theta}$ is elliptic and is first order in $B_{\theta}$, while the equation for $B_{\theta}$ can change type from elliptic to hyperbolic (and is also first order in $E_{\theta}$ ). This type change typically occurs in outer (low density) regions of the plasma and can be expressed in terms of relations between the applied frequency $\omega$ and the plasma and cyclotron frequencies of the electrons and ions. The question posed by Weitzner in [28] is the possible energy absorption by the plasma at applied frequencies on the order of the plasma frequency of an ionic species, called lower hybrid waves. The possible locations of heating occur at points $P$ of tangency between the lower hybrid type change surface and a level surface of the magnetic flux

Date: November 26, 2014.

2010 Mathematics Subject Classification. 35M10, 35M12, 35A15.

Key words and phrases. Mixed type PDE, spectral theory, variational methods, cold plasma model .

The authors have been partially supported by the Gruppo Nazionale per l'Analisi Matematica, la Probabilità e le loro Applicazioni (GNAMPA) of the Istituto Nazionale di Alta Matematica (INdAM).

Monticelli has also been supported by the GNAMPA project "Equazioni differenziali con invarianze in analisi globale" and by the MIUR project "Metodi variazionali e topologici nello studio di fenomeni nonlineari". 
function of the equilibrium magnetic field. In the axisymmetric setting, this situation is described by the tangency of two curves in the $(r, z)$ plane. Moreover, the type of degeneracy should be of Keldysh (and not Tricomi) type. This means that the second order differential operator at $P$ should admit a coordinate system $(\xi, \eta)$ in which the top order part looks like

$$
\left.\xi D_{\xi}^{2}+D_{\eta}^{2} \text { (as opposed to } D_{\xi}^{2}+\xi D_{\eta}^{2}\right) .
$$

The PDE in 1.1) is a local model of this situation in which $u=B_{\theta}$ and $(x, y)$ is a local coordinate system that straightens the level lines of the magnetic flux $\psi$ to be $x=$ constant.

While imposing the boundary condition $u=\phi$ on all of the boundary would seem physically natural, the presence of hyperbolicity should make the problem over-determined for classical solutions. This was first shown by Morawetz, Stevens and Weitzner [20] for the problem (1.1) minus the lower order term $u_{x} / 2$ and for suitable domains $\Omega$. The over-determinedness is proven by a suitable uniqueness theorem which shows that regular solutions are uniquely determined by their values on a proper subset of $\partial \Omega$ and exploits the technique developed by Morawetz [17] for equations of Tricomi type. On the other hand, Otway [21] has shown that the problem 1.1 with $\phi=0$ is weakly well-posed under suitable restrictions on $\Omega$ and $f$. This is accomplished by a global energy estimate technique which was developed by Morawetz and the authors (cf. [12] and [13]) for equations of Tricomi type. In order to perform this energy estimate technique, $\Omega$ will need to be star-shaped with respect to a suitable Lipschitz vector field which has a sink at a point on the boundary of $\Omega$, where this boundary point is precisely the point of possible plasma heating in the model equation (1.1).

Motivated by the relevance of the Dirichlet problem (1.1) to the question of plasma heating, we will address several basic mathematical issues and interpret their relevance in the context of the application. First, we will clarify and extend the solvability result of Otway by proving the well-posedness for weak solutions to the Dirichlet problem

$$
\begin{cases}L u:=-\left(K(x, y) u_{x}\right)_{x}-u_{y y}=f & \text { in } \Omega, \\ u=0 & \text { on } \partial \Omega,\end{cases}
$$

with $\Omega$ a bounded domain on which $K$ changes sign and is of the form

$$
K(x, y)=x-\sigma(y),
$$

where $\sigma$ satisfies

$$
\sigma \in C^{1}(\mathbb{R},[0,+\infty)), \sigma(0)=\sigma^{\prime}(0)=0 \text { and } \sigma \text { is not constant on any interval } I \subset \mathbb{R}
$$

and there exists $m \geq 1, C_{0}>0$ and $\delta_{0}>0$ such that

$$
\sigma(y) \leq C_{0}|y|^{m} \quad \text { if }|y|<\delta_{0},
$$

and

$$
m \sigma(y) \geq y \sigma^{\prime}(y) .
$$

We notice that conditions (1.5) - 1.7) allow for the Weitzner model $\sigma(y)=y^{2}$. The zero locus of $K$ is given by the curve $\Sigma$ described by $x=\sigma(y)$ and lies in the half plane where $x \geq 0$. One has $\Sigma$ tangent to the $y$ axis in the origin, the equation is of Keldysh type there, and the third condition in 1.5 ensures that the equation can be of Keldysh type only at isolated points (away from which it will be of Tricomi type). This condition will also be used in the spectral theory we develop for $L$ (see Theorem 3.6). Condition (1.6) allows for any order of degeneration compatible with the tangency of $\Sigma$ and the $y$-axis in the origin. The conditions 1.6 - 1.7) will be used in a multiplier argument to obtain the necessary global energy estimates which ensure the weak well-posedness of the Dirichlet problem (see Theorem 3.3). 
Next, we will extend the result to allow for Dirichlet data $\phi$ which are the boundary traces of a natural subspace of the weighted Sobolev space in which the weak solutions live. This will be accomplished by the standard trick of representing the solution as the sum $u=u_{0}+\phi$ where $u_{0}$ solves an analogous problem with zero boundary data. While this means that one can reduce the problem to one with homogeneous boundary data, the extension is relevant since the admissible local neighborhood $\Omega$ carries geometric restrictions and hence in general its boundary will not coincide with a level set of $u=B_{\theta}$.

We will then establish a spectral theory with weights for the problem which in turn will yield a variational characterization of the weak solutions, along the same lines of what the authors have accomplished for Tricomi type equations in [13] and [15. The solutions are saddle points of a naturally associated functional suggested by the divergence form of the differential operator $\operatorname{div}\left(K(x, y) D_{x}, D_{y}\right)$ and the solution space is the weighted Sobolev space corresponding to the natural domain of definition of the functional. This characterization proves that the problem is mathematically natural, despite the daunting difficulties presented by its overdetermined character with respect to classical solutions. A basic issue raised by Weitzner is what kinds of boundary conditions can yield well posed problems. For those models with a variational structure, the imposition of the Dirichlet condition on the entire boundary is essential for the variational characterization. Moreover, such a characterization also opens the possibility of a direct variational approach to prove the existence of solutions in those model situations in which the energy methods we employ fail to produce a proof of existence. Such a direct approach would most probably rely on suitably adapted minimax methods for strongly indefinite functionals (see Rabinowitz [25]).

The variational characterization takes the following form: if $\Omega$ and $K$ are suitable and if $f \in L^{2}(\Omega)$ then there exists a (unique) weak solution $u \in H_{0}^{1}(\Omega ; K)$ to the Dirichlet problem 1.3 if and only if $u$ is a critical point of the functional

$$
J(u)=\frac{1}{2} \int_{\Omega}\left(K(x, y) u_{x}^{2}+u_{y}^{2}\right) d x d y-\int_{\Omega} f u d x d y
$$

naturally associated to the equation. In addition, any critical point must be unique and is a saddle point, where the unique critical level will be described in terms of global extrema of the functional restricted to appropriate infinite dimensional subspaces. The notion of suitability of $\Omega$ and $K$ as well as the definitions of the weighted Sobolev space $H_{0}^{1}(\Omega ; K)$ and weak solutions will be recalled in Sections 2 and 3, along with other basic notions. By the solvability result to be presented herein (which generalizes that of Otway [21]), one knows that such a weak solution does indeed exist, provided that $f$ belongs to the subspace $L^{2}\left(\Omega ;|K|^{-1}\right)$ of $L^{2}(\Omega)$.

As already noted, this variational characterization depends strongly on an appropriate spectral theory with weights, which concerns the following weighted eigenvalue problem associated to $L$ :

$$
\begin{cases}-\left(K(x, y) u_{x}\right)_{x}-u_{y y}=\lambda W u & \text { in } \Omega, \\ u=0 & \text { on } \partial \Omega,\end{cases}
$$

where $W$ is any non-negative weight function satisfying $0<W \leq C_{W}|K|$ a.e. in $\Omega$ for some constant $C_{W}$. Armed with the aforementioned solvability theory, the spectral theory depends on two ingredients. In order to get started, one exploits the compactness of the solution operator (acting on the appropriate weighted version of $L^{2}(\Omega)$ ). This compactness depends on a suitable compact embedding result (see Lemma 3.7), which operates in a more general context and may prove to be of independent interest. The second ingredient involves the strongly indefinite nature of the quadratic form associated to the divergence form operator $L$. This indefinite nature strongly influences the spectral theory in which one has a doubly infinite sequence of eigenvalues (tending to $\pm \infty$ ).

A variational characterization of the problem (1.8) will also be given here as will variational characterizations of weak solutions of nonlinear Dirichlet problems of the form 


$$
\begin{cases}-\left(K(x, y) u_{x}\right)_{x}-u_{y y}=W g(x, y, u)+f(x, y) & \text { in } \Omega, \\ u=0 & \text { on } \partial \Omega,\end{cases}
$$

where $g: \Omega \times \mathbb{R} \rightarrow \mathbb{R}$ is a Carathéodory function, which is Lipschitz in $u$ uniformly with respect to $(x, y) \in \Omega$, and $f \in L^{2}(\Omega)$. We note that for Tricomi type equations, problems of the form 1.9 were studied using bifurcation, topological and dual variational methods in [13, [14, which is also possible in the present context. Instead of repeating the entire sequence of possible results, we will give two fixed point approaches to prove existence. The first approach uses the Leray-Schauder Principle and is applied to the problem (1.9) with $f=0$. The second approach uses the Contraction Mapping Principle and allows for nonlinear dependence also in the gradient of $u$; that is

$$
\begin{cases}-\left(K(x, y) u_{x}\right)_{x}-u_{y y}=W g\left(x, y, u, u_{x}, u_{y}\right) & \text { in } \Omega \\ u=0 & \text { on } \partial \Omega\end{cases}
$$

This last problem is then reconnected to plasma heating problem by way of an admissibility criterion for lower order terms in the linear model equations (see Corollary 5.4).

In Section 2, we recall the relevant background and notations, including the precise definitions of the function spaces in which we will operate. In Section 3 after giving the precise definition of weak solutions, we treat the questions of linear solvability and spectral theory, which will be reduced to three new technical results, which will be proven in Sections 6 and7. In Section 4, we will present the variational characterizations of weak solutions for the problems (1.3), (1.8) and (1.9). Finally, in Section 5 we will present the nonlinear existence results via fixed point methods, including that for the problem 1.10 .

We conclude this introduction with a few additional remarks about the Dirichlet problem for mixed type equations and about the cold plasma heating problem. As previously noted, mixed type equations in two independent variables will take one of the two canonical forms 1.2 . Beginning with the work of Frankl' [10], Tricomi type equations have long been associated with transonic potential flow in nozzles and about airfoils by way of a hodograph transformation (see also the modern survey of Morawetz [19]). Keldysh type equations are also known to play an important role in fluid mechanics as developed by Čanić and Keyfitz for the transonic small disturbance equation [3] and in Riemann problems for 2-D conservation laws [4] (see [1] and [2] for a discussion of singular and smooth solutions to the relevant associated degenerate elliptic Dirichlet problem). The study of the model equation (1.1) for wave propagation in plasmas appears to originate in Piliya and Fedorov [24]. For this model equation and the generalizations considered here, we have provided a solid mathematical formulation for its further study. More precisely, we have extended the class of equations to allow for arbitrary orders of degeneracy $m \geq 1$, provided an organic spectral theory with natural weights, and given variational characterizations of solutions. We have allowed for nonhomogenous boundary data and lower order terms in the equation which are important for the applications. In particular, the vanishing of the lower order coefficients when $K$ vanishes (see Corollary 5.4) appears to be compatible with the real lower order terms in the application (see Section 5 of [27]). In addition, we notice that for weak solutions we recover all of our recent results for Tricomi type equations, notwithstanding the substantial differences between the two forms in 1.2 . Our suspicion is that a fundamental role is played by the fact that the equation (1.1) and its generalizations display Keldysh type at only isolated points. One should expect substantial differences when examining higher order regularity of the weak solutions. We hope that the theory presented here will be supplemented by an adequate regularity theory and that it may prove useful for obtaining reliable estimates for the energy absorption at points of possible plasma heating (an important question which remains open as noted recently by Weitzner in Section 4 of [29]). Equations of mixed Tricomi and Keldysh forms have also been studied recently by Chen [5, although the equations considered there are not in divergence form and hence do not have a natural variational structure in general. 


\section{Notation and preliminary notions}

In all that follows, $K: \mathbb{R}^{2} \rightarrow \mathbb{R}$ will be of of the form (1.4) with $\sigma: \mathbb{R} \rightarrow[0,+\infty)$ satisfying (1.5) and

$$
\Sigma:=\{(x, y): x=\sigma(y)\}
$$

will denote the degeration set where $K$ vanishes. We will consider $\Omega \subset \mathbb{R}^{2}$ a mixed domain; that is, a bounded domain such that

$$
\Omega^{ \pm}:=\{(x, y) \in \Omega: \pm K>0\} \neq \emptyset .
$$

Notice that the differential operator defined by $L u=-\operatorname{div}\left(K u_{x}, u_{y}\right)$ will be of mixed elliptic-hyperbolic type in $\Omega$ and that the type change interface $\{K=0\}$ has measure zero. For $W$ a non-negative and measurable function and $\mathcal{M}(\Omega)$ the set of Lebesgue measurable functions on $\Omega$, we define the weighted Lebesgue space

$$
L^{2}(\Omega ; W):=\left\{f \in \mathcal{M}(\Omega): W^{1 / 2} f \in L^{2}(\Omega)\right\},
$$

which will be equipped with its natural norm

$$
\|f\|_{L^{2}(\Omega ; W)}=\left[\int_{\Omega} W f^{2} d x d y\right]^{1 / 2} .
$$

The weak solutions we treat will belong to the weighted Sobolev space $H_{0}^{1}(\Omega ; K)$ defined as the completion of $C_{0}^{1}(\Omega)$ with respect to the norm

$$
\|u\|_{H^{1}(\Omega ; K)}:=\left[\int_{\Omega}\left(|K| u_{x}^{2}+u_{y}^{2}+u^{2}\right) d x d y\right]^{1 / 2} .
$$

From [16], one knows that $C_{0}^{\infty}(\Omega)$ is dense in $H_{0}^{1}(\Omega ; K)$ and that one has a Poincaré inequality: there exists $C_{P}=C_{P}(\Omega, K)>0$ such that

$$
\|u\|_{L^{2}(\Omega)}^{2} \leq C_{P}^{2} \int_{\Omega}\left(|K| u_{x}^{2}+u_{y}^{2}\right) d x d y, \quad u \in H_{0}^{1}(\Omega ; K),
$$

so that an equivalent norm on $H_{0}^{1}(\Omega ; K)$ is given by

$$
\|u\|_{H_{0}^{1}(\Omega ; K)}:=\left[\int_{\Omega}\left(|K| u_{x}^{2}+u_{y}^{2}\right) d x d y\right]^{1 / 2} .
$$

We remark that elements of $H_{0}^{1}(\Omega ; K)$ may fail to admit a weak gradient in $\left[L^{2}(\Omega)\right]^{2}$ and hence some care must be given to the direct interpretation of the integral expressions for the norms. However, the weak gradients are well behaved away from the degeneration set $\Sigma$ given by 2.1 and there is a notion of weak quasi-gradient which allows one to manipulate easily such integral expressions. See Section 3 of [16] for a discussion of this technical point. The dual space to $H_{0}^{1}(\Omega ; K)$ will be denoted by $H^{-1}(\Omega ; K)$ and will be equipped with its negative norm in the sense of Lax

$$
\|w\|_{H^{-1}(\Omega ; K)}:=\sup _{0 \neq \varphi \in H_{0}^{1}(\Omega ; K)} \frac{|\langle w, \varphi\rangle|}{\|\varphi\|_{H_{0}^{1}(\Omega ; K)}} .
$$

\section{Linear solvability and spectral theory}

We begin by noting that $\mathcal{B}: H_{0}^{1}(\Omega, K) \times H_{0}^{1}(\Omega, K) \rightarrow \mathbb{R}$ defined by

$$
\mathcal{B}(u, v)=\int_{\Omega}\left(K u_{x} v_{x}+u_{y} v_{y}\right) d x d y, \quad \forall u, v \in H_{0}^{1}(\Omega ; K)
$$

is a continuous bilinear form associated to $L=-\operatorname{div}\left(K D_{x}, D_{y}\right)$. The notion of weak solution for the linear Dirichlet problem 1.3 with homogeneous boundary data is the following. 
Definition 3.1. For $f \in L^{2}(\Omega), u \in H_{0}^{1}(\Omega ; K)$ is called a weak solution of the Dirichlet problem [1.3] if

$$
\int_{\Omega}\left(K u_{x} v_{x}+u_{y} v_{y}\right) d x d y=\int_{\Omega} f v d x d y, \quad \forall v \in H_{0}^{1}(\Omega ; K) .
$$

In the case of non homogeneous boundary data $\phi \in H^{1}(\Omega ; K)$ will say that $u \in H^{1}(\Omega, K)$ is a weak solution of

$$
\begin{cases}L u:=-\left(K(x, y) u_{x}\right)_{x}-u_{y y}=f & \text { in } \Omega, \\ u=\phi & \text { on } \partial \Omega,\end{cases}
$$

provided that $u-\phi \in H_{0}^{1}(\Omega ; K)$ and $(3.2)$ holds. When we add lower order terms to $L$ or consider nonlinear variants, the obvious analogous notions of weak solution will be used. We note that if the boundary $\partial \Omega$ is reasonable, say Lipschitz, then the boundary condition $u=0$ holds in the trace sense (at least away from the points where $K=0$ ).

Given that $L$ is formally self adjoint, the existence of a unique weak solution $u \in H_{0}^{1}(\Omega ; K)$ to the linear Dirichlet problem 1.3 for each $f \in L^{2}\left(\Omega ;|K|^{-1}\right)$ is equivalent to the validity of a suitable apriori estimate valid on $\Omega$. We encode this estimate in the following definition.

Definition 3.2. We say that $\Omega$ is admissibile for $K$ if there exists $C>0$ such that

$$
\|u\|_{L^{2}(\Omega ;|K|)} \leq C|| L u \|_{H^{-1}(\Omega ; K)}, \quad u \in H_{0}^{1}(\Omega ; K) .
$$

We recall that $L^{2}\left(\Omega ;|K|^{ \pm 1}\right)$ are the weighted Lebesgue spaces 2.3 with weights $W=|K|^{ \pm 1}$. The fact that 3.3 characterizes the well-posedness was first shown for Tricomi type equations in [12] and used by Otway [21] for the model equation 11.1. Hence, given $K$ the real work to do is to find classes of domains $\Omega$ for which the estimate $(3.3)$ can be proven. Our first result is the following theorem.

Theorem 3.3. Let $K$ be defined by (1.4) with $\sigma$ satisfying (1.5), 1.6) and (1.7). Let $\Omega$ satisfy

$$
\bar{\Omega} \backslash\{(x, y): x>0\}=(0,0)=O
$$

and

$$
\Omega \text { is star-shaped with respect to the flow of the vector field } V=(-\beta x,-y) \text { for some } \beta>m+1 \text {. }
$$

Then one has the admissibility estimate (3.3) and hence for every $f \in L^{2}\left(\Omega ;|K|^{-1}\right)$ there exists a unique weak solution $u \in H_{0}^{1}(\Omega, K)$ to the Dirichlet problem [1.3) in the sense of [3.2].

The proof of this theorem which involves only minor adjustments of known results will be sketched in Section 6. For nonhomogeneous boundary data, one obtains easily the following result whose proof is standard.

Corollary 3.4. Let $\Omega$ and $K$ be as in Theorem 3.3 Let $\phi \in H^{1}(\Omega ; K)$ be such that there exists $F_{\phi} \in$ $L^{2}\left(\Omega ;|K|^{-1}\right)$ with

$$
\mathcal{B}(\phi, v)=\int_{\Omega} F_{\phi} v d x d y, \quad \forall v \in H_{0}^{1}(\Omega ; K),
$$

where $\mathcal{B}$ is the bilinear form 3.1 (which remains bounded on $H^{1}(\Omega ; K) \times H^{1}(\Omega ; K)$ ). Then there exists a unique $u \in H^{1}(\Omega ; K)$ satisfying $\left[3.2\right.$ and $u-\phi \in H_{0}^{1}(\Omega ; K)$.

We remark that, of course, the admissible data $\phi$ are boundary traces of all possible weak solutions to the PDE with source terms in $L^{2}\left(\Omega ;|K|^{-1}\right)$. The singularity in the weight $|K|^{-1}$ forces these sources to vanish in some sense along the degeneracy curve $\Sigma$ (where $K=0$ ). Examples of admissible $\phi$ are constants, polynomials in $K$ of degree at least three and more generally any $\phi \in C^{2}(\Omega)$ such that

$$
\int_{\Omega} \frac{|L \phi|^{2}}{|x-\sigma(x)|} d x d y<+\infty .
$$


For linear equations with lower order terms, solvability results will be obtained as a by-product of considering nonlinear perturbations of the problem.

We now turn our attention to spectral theory. Taking our cue from what happens for Tricomi type equations [13, if $\Omega$ is admissible for $K$ (i.e., the apriori estimate 3.3 holds), then one has a continuous solution operator

$$
S: L^{2}\left(\Omega ;|K|^{-1}\right) \rightarrow H_{0}^{1}(\Omega ; K)
$$

that is, $u=S(f)$ is the unique weak solution of the Dirichlet problem: $L u=f$ in $\Omega$ and $u=0$ on $\partial \Omega$. Notice that $S$ is not an operator on a fixed Hilbert space since $H_{0}^{1}(\Omega ; K) \not \subset L^{2}\left(\Omega ;|K|^{-1}\right)$. However, composing $S$ with a suitable multiplication operator yields a bounded linear operator on a larger space $L^{2}(\Omega ; W)$ which contains $H_{0}^{1}(\Omega ; K)$. For this reason, one introduces the following class of weight functions.

Definition 3.5. For $\Omega$ a bounded domain and $K$ fixed, define $\mathcal{W}$ the class of weight functions controlled by $|K|$ as the set of all $W \in L^{1}(\Omega)$ such that there exists a constant $C_{W}>0$ for which

$$
0<W \leq C_{W}|K| \text { a.e. on } \Omega \text {. }
$$

Notice that $W \in L^{\infty}(\Omega)$ for $\Omega$ bounded since $K \in C^{0}\left(\mathbb{R}^{2}\right)$. Notice also that $W$ vanishes on the vanishing set of $K$ if $W \in C^{0}(\Omega)$. For $\Omega$ bounded, simple examples include $W=|K|^{p}$ with $p \geq 1$. Composing the solution operator (3.6) with the multiplication operator $M_{W}$ (defined by $M_{W} f:=W f$ ) yields a continuous linear operator

$$
T:=S \circ M_{W}: L^{2}(\Omega ; W) \rightarrow H_{0}^{1}(\Omega ; K)
$$

which is a compact self-adjoint operator on $L^{2}(\Omega ; W)$ provided that

$$
H_{0}^{1}(\Omega ; K) \text { is compactly embedded in } L^{2}(\Omega) .
$$

The spectrum of $T$ is thus comprised of $\{0\}$ (which is not an eigenvalue) plus real eigenvalues of finite multiplicity. Using a standard mini-max procedure, one has a doubly infinite sequence of weighted eigenvalues $\left\{\lambda_{k}^{ \pm}\right\}_{k \in \mathbb{N}}$ provided that the quadratic form $\mathcal{Q}: H_{0}^{1}(\Omega ; K) \rightarrow \mathbb{R}$ defined by

$$
\mathcal{Q}(u):=\mathcal{B}(u, u)
$$

is strongly indefinite in the sense that for each $n \in \mathbb{N}$ there exists an orthogonal set $E_{n}=\left\{w_{k}^{+}\right\}_{k=1}^{n} \cup\left\{w_{k}^{-}\right\}_{k=1}^{n}$ in $H_{0}^{1}(\Omega ; K)$ such that

$$
\lim _{t \rightarrow+\infty} \mathcal{Q}\left(t w_{k}^{ \pm}\right)= \pm \infty, \quad k=1, \ldots n .
$$

For a mixed domain $\Omega$ and $K$ of the form (1.4) with $\sigma$ satisfying $(1.5)$, one always has the needed compactness (3.8) and the strongly indefinite property $(3.9)$ and hence one obtains the following result (analogous to Theorem 3.6 of [13] for equations of Tricomi type).

Theorem 3.6. Let $\Omega$ be a mixed domain which is admissible for $K$ of the form (1.4) with $\sigma$ satisfying (1.5) and let $W$ satisfy (3.7). Then $H_{0}^{1}(\Omega ; K)$ admits a basis $\mathcal{H}=\left\{u_{k}^{ \pm}\right\}_{k \in \mathbb{N}}$ of weak eigenfunctions to the problem 1.8 corresponding to a doubly infinite sequence of nonzero eigenvalues $\left\{\lambda_{k}^{ \pm}\right\}_{k \in \mathbb{N}}$ (counted with their finite multiplicity) such that

$$
\lambda_{k}^{+}>0, \quad \lambda_{k}^{+} \nearrow+\infty, \quad \lambda_{k}^{-}<0, \quad \lambda_{k}^{-} \searrow-\infty .
$$

Moreover, the basis $\mathcal{H}$ consists of functions which are orthogonal in $L^{2}(\Omega ; W)$ and which are $\mathcal{B}$-orthogonal in $H_{0}^{1}(\Omega ; K)$, where $\mathcal{B}: H_{0}^{1}(\Omega ; K) \times H_{0}^{1}(\Omega ; K) \rightarrow \mathbb{R}$ is the continuous bilinear defined in (3.1); that is,

$$
\mathcal{B}\left(u_{j}^{+}, u_{k}^{+}\right)=0=\mathcal{B}\left(u_{j}^{-}, u_{k}^{-}\right), \quad \forall j \neq k \in \mathbb{N}, \quad \mathcal{B}\left(u_{j}^{+}, u_{k}^{-}\right)=0, \quad \forall j, k \in \mathbb{N} .
$$


Notice that we are assuming the admissibility estimate (3.3) which ensures the solvability. By Theorem 3.3. one has this estimate if one also assumes (1.6), (1.7), 3.4) and (3.5). Given the admissibility, it remains only to verify the compactness and strongly indefinite properties when $\Omega$ and $\sigma$ satisfy $(2.2)$ and 1.5 . We will show that these two properties hold in a much more general context. For future use, we formulate them as two lemmas.

The compact embedding property (3.8) will be treated in the context of compact embeddings of Sobolev spaces with matrix weights into Lebesgue spaces. We will denote by $\operatorname{Sym}_{N}^{+}(\mathbb{R})$ the space of real symmetric $N \times N$ matrices such that the associated quadratic form is non-negative definite. Given any measurable function $A: \Omega \rightarrow \operatorname{Sym}_{N}^{+}(\mathbb{R})$, we define $W_{0}^{1, p}(\Omega ; A)$ as the completion of $C_{0}^{1}(\Omega)$ with respect to the norm

$$
\|u\|_{W_{0}^{1, p}(\Omega ; A)}=\left[\int_{\Omega}\langle A D u, D u\rangle^{p / 2} d x\right]^{1 / p}=\left[\int_{\Omega}\left\langle A^{1 / 2} D u, A^{1 / 2} D u\right\rangle^{p / 2} d x\right]^{1 / p} .
$$

We note that our weighted space $H_{0}^{1}(\Omega ; K)$ is precisely $W_{0}^{1,2}(\Omega ; A)$ for the matrix valued function

$$
A\left(x_{1}, x_{2}\right)=\left[\begin{array}{cc}
1 & 0 \\
0 & \left|K\left(x_{1}, x_{2}\right)\right|
\end{array}\right]=\left[\begin{array}{cc}
1 & 0 \\
0 & \left|x_{2}-\sigma\left(x_{1}\right)\right|
\end{array}\right],
$$

with $\left(x_{1}, x_{2}\right)=(y, x)$. The following compact embedding lemma will be proven in Section 7 by applying a general result of Danielli [6] for weighted Sobolev spaces associated to weak sub-Riemannian structures underlying our anisotropic setting. In what follows, for each $j \in\{1, \ldots, N\}$, we will denote by $\Pi_{j}: \mathbb{R}^{N} \rightarrow \mathbb{R}^{j}$ the canonical projection onto the first $j$ components.

Lemma 3.7. Let $\Omega \subset \mathbb{R}^{N}$ be a bounded domain and let $\Omega_{0}$ be an open neighborhood of $\bar{\Omega}$. Given any family of weights $\left\{\Lambda_{j}\right\}_{j=1}^{N} \subset C^{1}\left(\Omega_{0},[0,+\infty)\right)$ satisfying

$$
\begin{gathered}
\Lambda_{j}=\Lambda_{j}\left(x_{1}, \ldots, x_{j}\right), \quad j=1, \ldots, N ; \\
\Lambda_{1}>0 \text { on } \Omega_{0} ;
\end{gathered}
$$

and for each fixed $j=1, \ldots, N$ and $\left(z_{1}, \ldots, z_{j}\right) \in \Pi_{j}(\Omega)$ such that $\Lambda_{j}\left(z_{1}, \ldots, z_{j}\right)=0$ :

$$
S_{\varepsilon}\left(z_{1}, \ldots, z_{j}\right) \text { contains no arc of a } C^{1} \text { curve of positive length if } \varepsilon>0 \text { is sufficiently small }
$$

where

$$
S_{\varepsilon}\left(z_{1}, \ldots, z_{j}\right):=\left\{\left(x_{1}, \ldots, x_{j-1}\right) \in \prod_{k=1}^{j-1}\left[z_{k}, z_{k}+\varepsilon\right]: \Lambda_{j}\left(x_{1}, \ldots, x_{j-1}, z_{j}\right)=0\right\} .
$$

Then one has the following compact embedding results.

a) For each $p \in[1, \infty)$

$$
W_{0}^{1, p}\left(\Omega, \Lambda^{2}\right) \hookrightarrow \hookrightarrow L^{p}(\Omega)
$$

where $W_{0}^{1, p}\left(\Omega, \Lambda^{2}\right)$ is the completion of $C_{0}^{1}(\Omega)$ with respect to the norm 3.10 with $A=\Lambda^{2}$ and $\Lambda$ is the diagonal matrix with entries $\Lambda_{1}, \ldots \Lambda_{N}$.

b) If $A: \Omega \rightarrow \operatorname{Sym}_{N}^{+}(\mathbb{R})$ is measurable and if there exists $C>0$ such that

$$
C\left\langle\Lambda^{2}(x) \xi, \xi\right\rangle \leq\langle A(x) \xi, \xi\rangle \text { for a.e. } x \in \Omega \text { and each } \xi \in \mathbb{R}^{N},
$$

then for each $p \in[1, \infty)$

$$
W_{0}^{1, p}(\Omega ; A) \hookrightarrow \hookrightarrow L^{p}(\Omega)
$$

In order to show the strongly indefinite property $(3.9)$ on mixed domains, we will provide a general result for indefinite quadratic forms which can be applied to second order PDEs of mixed elliptic-hyperbolic type. We denote by $\operatorname{Sym}_{N}(\mathbb{R})$ the space of real symmetric $N \times N$ matrices. 
Lemma 3.8. Let $\Omega$ be an open subset of $\mathbb{R}^{N}$ and let $Q: \Omega \rightarrow \operatorname{Sym}_{N}(\mathbb{R})$ be continuous.

a) If there exist $B_{r}\left(x_{0}\right) \subset \subset \Omega, \zeta \in \mathbb{R}^{N}$ with $|\zeta|=1$ and $\delta>0$ such that

$$
\langle Q(x) \zeta, \zeta\rangle \geq \delta>0, \quad \forall x \in B_{r}\left(x_{0}\right),
$$

then for each $n \in \mathbb{N}$ there exists a collection of functions $\left\{w_{1}, \ldots, w_{n}\right\} \subset C_{0}^{\infty}(\Omega)$ having mutually disjoint supports in $B_{r}\left(x_{0}\right)$ such that

$$
\int_{\Omega}\left\langle Q(x) D w_{j}, D w_{j}\right\rangle d x>0, \quad j=1, \ldots, n .
$$

b) If there exist $B_{r}\left(x_{0}\right) \subset \subset \Omega, \zeta \in \mathbb{R}^{N}$ with $|\zeta|=1$ and $\delta>0$ such that

$$
\langle Q(x) \zeta, \zeta\rangle \leq-\delta<0, \quad \forall x \in B_{r}\left(x_{0}\right),
$$

then for each $n \in \mathbb{N}$ there exists a collection of functions $\left\{w_{1}, \ldots, w_{n}\right\} \subset C_{0}^{\infty}(\Omega)$ having mutually disjoint supports in $B_{r}\left(x_{0}\right)$ such that

$$
\int_{\Omega}\left\langle Q(x) D w_{j}, D w_{j}\right\rangle d x<0, \quad j=1, \ldots, n .
$$

This simple auxiliary result will also be proven in Section 7 and just involves the construction of suitable bump functions depending on $\zeta, B_{r}\left(x_{0}\right)$ and $\delta$.

Proof of Theorem [3.6. As mentioned above, the body of the proof is identical to that of Theorem 3.6 of [13] provided one has the admissibility estimate (3.3), the compact embedding $(3.8)$ and the strongly indefinite property $(3.9)$ for the mixed domain $\Omega$. Briefly the idea is the following. Given the three ingredients mentioned above, one combines the strongly indefinite property with the standard mini-max construction (see Lemma 3.4 of [13]) to give a variational construction of eigenvalues for the compact operator

$$
T:=S \circ M_{W}: L^{2}(\Omega ; W) \rightarrow H_{0}^{1}(\Omega ; K) \hookrightarrow \hookrightarrow L^{2}(\Omega ; W) .
$$

One obtains a doubly infinite sequence of (nonzero) eigenvalues $\left\{\mu_{k}^{ \pm}\right\}_{k \in \mathbb{N}}$ for the operator $T$ with corresponding eigenfunctions $\left\{u_{k}^{ \pm}\right\}_{k \in \mathbb{N}} \subset H_{0}^{1}(\Omega ; K)$. These are the weak eigenfunctions sought for $L$ where $\lambda_{k}^{ \pm}=1 / \mu_{k}^{ \pm}$. The proof of these facts is identical to that in the Tricomi type case (see Theorem 3.6 of [13]).

In order to prove the compactness property (3.8), we apply part b) of Lemma 3.7 with the matrix weight $A$ corresponding to $H_{0}^{1}(\Omega ; K)$ (as given by (3.11) ) and select $\Lambda=A^{2}$; that is

$$
\Lambda_{1}\left(x_{1}\right)=1 \text { and } \Lambda_{2}\left(x_{1}, x_{2}\right)=K^{2}\left(x_{1}, x_{2}\right)=\left(x_{2}-\sigma\left(x_{1}\right)\right)^{2} .
$$

Clearly $\Lambda \in C^{1}\left(\bar{\Omega}, \operatorname{Sym}_{2}^{+}(\mathbb{R})\right)$ and one has $(3.12)$ and (3.13) since $\Lambda_{1}=\Lambda_{1}\left(x_{1}\right) \equiv 1>0$. One has also (3.14). Indeed, if $0=\Lambda_{2}\left(z_{1}, z_{2}\right)=\left(z_{2}-\sigma\left(z_{1}\right)\right)^{2}$ for some $\left(z_{1}, z_{2}\right) \in \Omega$, the existence of a small $\varepsilon>0$ such that

$$
S_{\varepsilon}\left(z_{1}, z_{2}\right)=\left\{x_{1} \in\left[z_{1}, z_{1}+\varepsilon\right]: z_{2}=\sigma\left(x_{1}\right)\right\}
$$

contains an arc of a $C^{1}$ curve of positive length would contradict the fact that $\sigma$ is not constant on any interval (as required by (1.5)). Finally, the needed lower bound 3.16 is

$$
C\left(\xi_{1}^{2}+|K|^{4} \xi_{2}^{2}\right) \leq \xi_{1}^{2}+|K| \xi_{2}^{2},
$$

which holds for $C^{-1}=\sup _{\Omega}|K|^{3}+1$.

In order to prove the strongly indefinite property $(3.9)$, we apply Lemma 3.8 with $Q$ given by

$$
Q\left(x_{1}, x_{2}\right)=\left[\begin{array}{cc}
1 & 0 \\
0 & K\left(x_{1}, x_{2}\right)
\end{array}\right]=\left[\begin{array}{cc}
1 & 0 \\
0 & x_{2}-\sigma\left(x_{1}\right)
\end{array}\right],
$$

Since $\Omega$ is a mixed domain and $K$ is continuous and changes sign, if $x_{0}$ belongs to the elliptic region $\Omega^{+}$, which is open, one can choose a ball $B^{+}=B_{r}\left(x_{0}\right)$ lying in $\Omega^{+}$. On that ball, take $\zeta=(0,1)$ and $\delta=\inf _{B^{+}} K>0$. 
Similarly, the hyperbolic region $\Omega^{-}$is also open and choose again $\zeta=(0,1)$ but take $-\delta=\sup _{B^{-}} K<0$. The family $\left\{w_{1}^{ \pm}, \ldots, w_{n}^{ \pm}\right\}$clearly gives an orthogonal set in $H_{0}^{1}(\Omega ; K)$ and one has the needed limits in (3.9).

\section{Variational characterizations of solutions}

We now describe the variational characterizations for weak solutions to the Dirichlet problem (1.3) and perturbations of that problem. As mentioned in the introduction, the characterizations presuppose the validity of the solvability and spectral theory of the previous section. The following definition encodes all that we need to assume in order to ensure the needed ingredients.

Definition 4.1. Let $K(x, y)=x-\sigma(y)$ where $\sigma$ satisfies 1.5 . We call $(\Omega, \sigma)$ a Dirichlet pair for $L=$ $-\operatorname{div}\left(K D_{x}, D_{y}\right)$ if 2.2$),(3.3)$ and $(3.8)$ hold.

We recall that $(3.3)$ ensures the validity of the solvability Theorem 3.3 . The condition 2.2 and the form of $K$ ensures the strongly indefinite property (3.9) which combined with (3.8) then yields the spectral theory of Theorem 3.6. We have shown that $(\Omega, \sigma)$ is indeed a Dirichlet pair if one also assumes that $(\Omega, \sigma)$ satisfy (3.4, (3.5), (1.6) and (1.7).

In all that follows, $\mathcal{B}$ will be the bilinear form defined in 3.1 and we will exploit Theorem 3.6 in order to split the space $H_{0}^{1}(\Omega ; K)$ into $\mathcal{B}$-orthogonal subspaces, which will also be orthogonal with respect to the $L^{2}(\Omega ; W)$ inner product, and we will then consider the restrictions of the functionals and their differentials to those subspaces. More precisely, by defining $H^{ \pm}:=\operatorname{span}\left\langle u_{k}^{ \pm}: k \in \mathbb{N}\right\rangle$ one has $H_{0}^{1}(\Omega ; K)=H^{+} \oplus H^{-}$where the two subspaces are $\mathcal{B}$-orthogonal, but not orthogonal with respect to the inner product of $H_{0}^{1}(\Omega ; K)$. Let $P^{ \pm}: H_{0}^{1}(\Omega ; K) \rightarrow H^{ \pm}$be the natural projections onto those subspaces. For any $f \in L^{2}(\Omega)$, define the functionals

$$
J(u):=\frac{1}{2} \mathcal{B}(u, u)-\int_{\Omega} f u d x d y \text { and } J^{ \pm}=J \circ P^{ \pm} .
$$

It is clear that $J, J^{+}, J^{-}$are $C^{1}$ functionals on $H_{0}^{1}(\Omega ; K)$ and that

$$
J^{\prime}(u)[h]=\int_{\Omega}\left(K u_{x} h_{x}+u_{y} h_{y}\right) d x d y-\int_{\Omega} f h d x d y \quad \forall u, h \in H_{0}^{1}(\Omega ; K) .
$$

Hence weak solutions of the Dirichlet problem (1.3) are precisely the critical point(s) of $J$. The functional $J$ is strongly indefinite; that is, there exist infinite dimensional subspaces $H^{ \pm}$for which $J \rightarrow \pm \infty$ along all rays in $H^{ \pm}$. Recall that we have seen that the quadratic part $\mathcal{Q}(u)=\mathcal{B}(u, u)$ has this property. The following theorem includes a variational characterization of the unique weak solution of 1.3 of Theorem 3.3 for $f \in L^{2}\left(\Omega ;|K|^{-1}\right)$.

Theorem 4.2. Let $(\Omega, \sigma)$ be a Dirichlet pair and $f \in L^{2}(\Omega)$. Then

a) the following are equivalent:

i) $u \in H_{0}^{1}(\Omega ; K)$ is a weak solution of 1.3 ;

ii) $u$ is a critical point of the functional $J$ defined in 4.1);

iii) $u$ is a critical point of the functionals $J^{+}$and $J^{-}$defined in 4.1);

iv) $u$ is a global extremum of $J^{+}$and $J^{-}$in the sense that

$$
J^{+}(u)=J_{*}^{+}:=\inf _{h \in H} J^{+}(h) \quad \text { and } \quad J^{-}(u)=J_{*}^{-}:=\sup _{h \in H} J^{-}(h) ;
$$

b) $J$ is strongly indefinite, it does not have local minima or maxima and it has at most one critical point, which must be a saddle point. 
c) There is at most one critical level for $J$, which is finite and given by

$$
J^{\star}=J_{\star}=J_{*}^{+}+J_{*}^{-},
$$

where

$$
J^{\star}:=\inf _{v \in H^{+}} \sup _{w \in H^{-}} J(v+w) \quad \text { and } \quad J_{\star}:=\sup _{w \in H^{-}} \inf _{v \in H^{+}} J(v+w),
$$

and $J_{*}^{ \pm}$as defined in 4.2 .

d) If $f \in L^{2}\left(\Omega ;|K|^{-1}\right)$, then the critical level 4.3) is achieved by $u$ the unique weak solution of (1.3) which satisfies the properties in part a) of the theorem.

The proof of this theorem is identical to that of Theorem 2.8 of [15] for Tricomi type equation and hence will be omitted. However, to aid the reader perhaps a few remarks are in order. First, we note that

$$
J_{*}^{+}=\inf _{v \in H^{+}} J_{H_{H^{+}}}(v) \quad \text { and } \quad J_{*}^{-}=\sup _{w \in H^{-}} J_{H_{H^{-}}}(w),
$$

so that $(4.2)$ is equivalent to

$$
J\left(P^{+}(u)\right)=\inf _{v \in H^{+}} J_{\left.\right|_{H^{+}}}(v) \quad \text { and } \quad J\left(P^{-}(u)\right)=\sup _{w \in H^{-}} J_{\left.\right|_{H^{-}}}(w) .
$$

Second, one makes use of some basic relations between the critical levels $J^{\star}, J_{\star}$ defined in (4.4) and $J_{*}^{+}, J_{*}^{-}$ defined in $(4.2)$, where we note that

$$
J_{*}^{+} \in[-\infty,+\infty) \text { and } J_{*}^{-} \in(-\infty,+\infty] .
$$

Those relations are contained in Lemma 3.1 of [15, which we reproduce here without proof.

Lemma 4.3. If $(\Omega ; K)$ is a Dirichlet pair then

a) $J_{*}^{+}>-\infty$ or $J_{*}^{-}<\infty \Rightarrow J^{\star}=J_{\star}=J_{*}^{+}+J_{*}^{-}$;

b) $J^{\star}=J_{\star} \Rightarrow J_{*}^{+}>-\infty$ or $J_{*}^{-}<\infty$;

c) $J^{\star} \in \mathbb{R} \Leftrightarrow J_{\star} \in \mathbb{R} \Leftrightarrow J_{*}^{+}, J_{*}^{-} \in \mathbb{R}$.

Consult Section 3 of [15] for details.

Remark 4.4. The condition $f \in L^{2}\left(\Omega ;|K|^{-1}\right)$ is obviously not necessary in order to have a weak solution of (1.3), or equivalently for the critical level of $J$ to be achieved, as the following example shows.

Example 4.5. Let $\Omega \subset \mathbb{R}^{2}$ be a bounded domain satisfying $(2.2)$ and let $\Sigma=\left\{(x, y) \in \mathbb{R}^{2}: x=\sigma(y)\right\}$ be the degeneration set for $L=-\operatorname{div}\left((x-\sigma(y)) D_{x}, D_{y}\right)$ with $\sigma$ satisfying 1.5$)$. Fix $P=\left(x_{0}, y_{0}\right) \in \Omega$ with $x_{0}=\sigma\left(y_{0}\right)$ and fix $r>0$ such that $\bar{B}_{r}(P) \subset \Omega$. Define the function $u$ on $\bar{\Omega}$ by

$$
u(x, y):=y^{2} \eta(x, y),
$$

where $\eta \in C_{0}^{\infty}\left(\mathbb{R}^{2}\right)$ is non-negative, vanishes outside of $B_{r}(P)$ and satisfies $\eta \equiv 1$ in $B_{\frac{r}{2}}(P)$. Then $u \geq 0$ in $\Omega$ and is a classical (and hence weak) solution of 1.3 with

$$
f \in L^{2}(\Omega) \backslash L^{2}\left(\Omega ;|K|^{-1}\right) .
$$

Clearly $u \in C_{0}^{\infty}(\Omega)$ and $\operatorname{supp}(u) \subset B_{r}(P)$ so that $u \in H_{0}^{1}(\Omega ; K)$ and satisfies the boundary condition in the classical sense. Moreover, one has

$$
L u=(x-\sigma(y)) y^{2} \eta_{x x}+y^{2} \eta_{y y}+4 y \eta_{y}+2 \eta+y^{2} \eta_{x}:=f \quad \text { in } \Omega .
$$


Clearly $f \in C^{1}(\Omega)$ with support in $\bar{B}_{r}(P) \subset \Omega$ so that $f \in L^{2}(\Omega)$. On the other hand, $f \notin L^{2}\left(\Omega ;|K|^{-1}\right)$ with $K(x, y)=x-\sigma(y)$. Indeed, estimating from below on

$$
\left[x_{0}-\frac{r}{2 \sqrt{2}}, x_{0}+\frac{r}{2 \sqrt{2}}\right] \times\left[y_{0}-\frac{r}{2 \sqrt{2}}, y_{0}+\frac{r}{2 \sqrt{2}}\right] \subset \bar{B}_{\frac{r}{2}}(P) \subset B_{r}(P)
$$

where $\eta \equiv 1$ one has

$$
\|f\|_{L^{2}\left(\Omega ;|K|^{-1}\right)}^{2} \geq \int_{y_{0}-\frac{r}{2 \sqrt{2}}}^{y_{0}+\frac{r}{2 \sqrt{2}}} \int_{x_{0}-\frac{r}{2 \sqrt{2}}}^{x_{0}+\frac{r}{2 \sqrt{2}}} \frac{|f|^{2}}{|x-\sigma(y)|} d x d y=\int_{y_{0}-\frac{r}{2 \sqrt{2}}}^{y_{0}+\frac{r}{2 \sqrt{2}}} \int_{x_{0}-\frac{r}{2 \sqrt{2}}}^{x_{0}+\frac{r}{2 \sqrt{2}}} \frac{4}{|x-\sigma(y)|} d x d y .
$$

Since $1 /|x-\sigma(y)|$ is not integrable in $x \in\left[x_{0}-\frac{r}{2 \sqrt{2}}, x_{0}+\frac{r}{2 \sqrt{2}}\right]$ for almost every $y \in\left[y_{0}-\frac{r}{2 \sqrt{2}}, y_{0}+\frac{r}{2 \sqrt{2}}\right]$, by Tonelli's theorem, one concludes that $f \notin L^{2}\left(\Omega ;|K|^{-1}\right)$.

We now proceed to state a sequence of similar variational characterizations for weak solutions of various perturbations of the problem 1.3. These results mirror what was done in [15] for equations of Tricomi type and their proofs are identical. We begin by adding a non-trivial term of order zero; that is, we consdier

$$
\left\{\begin{array}{l}
-\left(K(x, y) u_{x}\right)_{x}-u_{y y}-\lambda W u=f \quad \text { in } \Omega, \\
u=0 \quad \text { on } \partial \Omega,
\end{array}\right.
$$

This problem is clearly related to the weighted eigenvalue problem 1.8$)$. We denote by $\sigma(L)$ the set of eigenvalues of $L$; that is, $\lambda \in \sigma(L)$ if there exists a nontrivial weak solution $u \in H_{0}^{1}(\Omega ; K)$ to the problem (1.8). We define the subspaces of $H_{0}^{1}(\Omega ; K)$

$$
\left\{\begin{array}{l}
H_{\lambda}^{-}=\operatorname{span}\left\langle u \in H_{0}^{1}(\Omega ; K): u \text { eigenfunction associated to an eigenvalue } \mu<\lambda\right\rangle \\
H_{\lambda}^{+}=\operatorname{span}\left\langle u \in H_{0}^{1}(\Omega ; K): u \text { eigenfunction associated to an eigenvalue } \mu>\lambda\right\rangle
\end{array}\right.
$$

and we consider the modified functional $J_{\lambda}(u): H_{0}^{1}(\Omega ; K) \rightarrow \mathbb{R}$ defined by

$$
J_{\lambda}(u)=\frac{1}{2} \mathcal{B}(u, u)-\frac{\lambda}{2} \int_{\Omega} W u^{2} d x d y-\int_{\Omega} f u d x d y \quad \forall u \in H_{0}^{1}(\Omega ; K) .
$$

We denote by $P_{\lambda}^{ \pm}$the natural projections of $H_{0}^{1}(\Omega ; K)$ onto the subspaces $H_{\lambda}^{ \pm}$and define $J_{\lambda}^{ \pm}:=J_{\lambda} \circ P_{\lambda}^{ \pm}$. The variational characterization for the problem 4.5 is provided by the following theorem.

Theorem 4.6. Let $(\Omega, \sigma)$ be a Dirichlet pair for $L$ and $f \in L^{2}(\Omega)$. Then

a) if $\lambda \notin \sigma(L)$, the function $u \in H_{0}^{1}(\Omega ; K)$ is a weak solution of 4.5 if and only if

$$
J_{\lambda}^{+}(u)=\inf _{h \in H} J_{\lambda}^{+}(h) \quad \text { and } \quad J_{\lambda}^{-}(u)=\sup _{h \in H} J_{\lambda}^{-}(h) ;
$$

b) if $\lambda \in \sigma(L)$ with associated eigenspace $E_{\lambda}$, the function $u \in H_{0}^{1}(\Omega ; K)$ is a weak solution of 4.5 if and only if (4.7) and the following condition hold

$$
\int_{\Omega} f v d x=0 \quad \forall v \in E_{\lambda} .
$$

Remark 4.7. Formulas similar to (4.4) and (4.3) hold also for $J_{\lambda}$. Moreover, if $f \in L^{2}\left(\Omega ; W^{-1}\right)$ then by repeating the argument of Theorem 2.3 of [14] for equations of Tricomi type, one obtains the following Fredholm alternative:

I) if $\lambda \notin \sigma(L)$, then problem (4.5) admits a unique weak solution, and thus $J_{\lambda}$ has a unique critical point, which is a saddle point;

II) if $\lambda \in \sigma(L)$ with associated eigenspace $E_{\lambda}$, then problem 4.5 admits weak solutions, and thus $J_{\lambda}$ has infinitely many critical points if and only if 4.8 holds. The critical points $u$ are saddle points which are degenerate in the sense that $J_{\lambda}^{\prime \prime}(u)[h, h]=0$ for each $h \in E_{\lambda}$, where $J_{\lambda}$ is clearly a $C^{2}$ functional. 
As noted in the proof of Theorem 3.6, the eigenvalues of $L$ were characterized variationally through the inverse operator $S=L^{-1}$. We are now able to provide a direct variational characterization of the eigenvalues and weak eigenfunctions in terms of the Rayleigh quotient

$$
\mathcal{R}(v):=\frac{\mathcal{B}(v, v)}{\int_{\Omega} W v^{2} d x d y}, \quad v \in H_{0}^{1}(\Omega ; K) .
$$

We begin with $\lambda_{1}^{ \pm}$.

Theorem 4.8. Let $(\Omega, \sigma)$ be a Dirichlet pair for $L$ and $\mathcal{R}(v)$ defined by 4.9$)$. Then

a) $\lambda_{1}^{+}=\inf _{v \in H^{+} \backslash\{0\}} \mathcal{R}(v) \quad$ and $\quad \lambda_{1}^{-}=\sup _{v \in H^{-} \backslash\{0\}} \mathcal{R}(v)$;

b) $u \in H_{0}^{1}(\Omega ; K)$ is a weak eigenfunction of problem 1.8 associated to $\lambda_{1}^{+}$if and only if $u \in H^{+}$and

$$
\mathcal{R}(u)=\inf _{v \in H^{+} \backslash\{0\}} \mathcal{R}(v) ;
$$

c) $u \in H_{0}^{1}(\Omega ; K)$ is a weak eigenfunction of problem (1.8) associated to $\lambda_{1}^{-}$if and only if $u \in H^{-}$and

$$
\mathcal{R}(u)=\sup _{v \in H^{-} \backslash\{0\}} \mathcal{R}(v) .
$$

Using the standard inductive device of removing the span of eigenfunctions corresponding to the eigenvalues previously constructed, one obtains sequentially the remaining eigenvalues ordered by increasing magnitude.

Finally, we turn our attention to variational characterizations for nonlinear perturbations; that is, for the problem (1.9). We assume that the nonlinearity $g=g(x, y, t): \Omega \times \mathbb{R} \rightarrow \mathbb{R}$ satisfies the Carathéodory conditions

$$
g \text { is measurable in }(x, y) \text { for each } t \in \mathbb{R} \text { and continuous in } t \text { for a.e. }(x, y) \in \Omega \text {. }
$$

This ensures the continuity of the Nemytski operators associated to $g$ and its primitives in $t$ (see the discussion in the next section). We will also assume the following condition: there exist $m, M \in \mathbb{R}$ such that

$$
m \leq \frac{g(x, y, t+r)-g(x, y, t)}{r} \leq M
$$

for every $t \in \mathbb{R}, r \in \mathbb{R} \backslash\{0\}$ and a.e. $(x, y) \in \Omega$. Condition 4.11) is equivalent to requiring that the function $g(x, y, t)$ is Lipschitz in $t \in \mathbb{R}$, uniformly for a.e. $(x, y) \in \Omega$. We consider the associated functional $I: H_{0}^{1}(\Omega ; K) \rightarrow \mathbb{R}$

$$
I(u)=\frac{1}{2} \mathcal{B}(u, u)-\int_{\Omega} W G(x, y, u)+f(x, y) u d x d y \quad \forall u \in H_{0}^{1}(\Omega ; K)
$$

where $G(x, y, t)=\int_{0}^{t} g(x, y, s) d s$. We will consider the subspaces $H_{m}^{-}$and $H_{M}^{+}$as defined in 4.6 with $\lambda=m$ and $\lambda=M$ respectively. Notice that $H_{m}^{-} \oplus H_{M}^{+} \subsetneq H_{0}^{1}(\Omega ; K)$ if $\sigma(L) \cap[m, M] \neq \emptyset$. We define

$$
\Lambda^{m}:=\max \{\lambda \in \sigma(L) \mid \lambda<m\}, \quad \Lambda_{M}:=\min \{\lambda \in \sigma(L) \mid \lambda>M\} .
$$

The variational characterization for the nonlinear Dirichlet problem 1.9$)$ is the following result.

Theorem 4.9. Let $(\Omega, K)$ be a Dirichlet pair for $L, g$ a nonlinearity satisfying 4.10 - 4.11) and $f \in L^{2}(\Omega)$. Then $I \in C^{1}\left(H_{0}^{1}(\Omega ; K), \mathbb{R}\right)$ and

a) $u \in H_{0}^{1}(\Omega ; K)$ is a weak solution of 1.9 if and only if $u$ is a critical point of the functional I defined by 4.12 ;

b) for every $u, h \in H_{0}^{1}(\Omega ; K)$ one has

$$
\frac{1}{2} \mathcal{B}(h, h)-\frac{M}{2}\|h\|_{L^{2}(\Omega ; W)}^{2} \leq I(u+h)-I(u)-I^{\prime}(u)[h] \leq \frac{1}{2} \mathcal{B}(h, h)-\frac{m}{2}\|h\|_{L^{2}(\Omega ; W)}^{2} ;
$$


c) if $u \in H_{0}^{1}(\Omega ; K)$ is a weak solution of 1.9 , then

$$
\begin{aligned}
& I(u+h) \leq I(u)+\frac{\Lambda^{m}-m}{2}\|h\|_{L^{2}(\Omega ; W)}^{2} \quad \text { for all } h \in H_{m}^{-}, \\
& I(u+h) \geq I(u)+\frac{\Lambda_{M}-M}{2}\|h\|_{L^{2}(\Omega ; W)}^{2} \quad \text { for all } h \in H_{M}^{+} .
\end{aligned}
$$

In particular, $u$ is a saddle point for $I$ and

$$
I(u)=\max _{h \in H_{m}^{-}} I(u+h)=\min _{h \in H_{M}^{+}} I(u+h) ;
$$

d) if $\sigma(L) \cap[m, M]=\emptyset$, then $u \in H_{0}^{1}(\Omega ; K)$ is a solution of 1.9 if and only if 4.13 holds.

Remark 4.10. Existence of weak solutions for nonlinear Dirichlet problems of the form (1.9) were considered in 14 for Tricomi type equations, but often without the requirement that $g(x, y, t)$ be Lipschitz in $t \in \mathbb{R}$. One could develop an analogous existence theory here, impose this additional Lipschitz requirement and then apply Theorem 4.9 to give a variational characterization of such solutions. That is, one can obtain examples where the critical points in Theorem 4.9 actually exist.

\section{Existence results for nonlinear problems}

In this section, we put the linear solvability and spectral theory to work in order to treat the question of existence of weak solutions to semilinear Dirichlet problems of the form $(1.9)$ with $f=0$ and $(1.10)$. We assume throughout that $K$ has the form (1.4); that is, $K(x, y)=x-\sigma(y)$ with $\sigma$ satisfying (1.5). Moreover we will assume that $(\Omega, \sigma)$ is a Dirichlet pair for $L=-\operatorname{div}\left(K D_{x}, D_{y}\right)$ as defined in Defintion 4.1 so that we indeed have solvability and spectral theory of Section 3 for weights $W$ belonging to the weight class $\mathcal{W}$ of Definition 3.7. This will allow us to set up a fixed point argument for the nonlinear problems by first inverting the differential operator $L$.

We begin by considering the problem

$$
\begin{cases}L u:=-\left(K(x, y) u_{x}\right)_{x}-u_{y y}=W g(x, y, u) & \text { in } \Omega \\ u=0 & \text { on } \partial \Omega\end{cases}
$$

where $g: \Omega \times \mathbb{R} \rightarrow \mathbb{R}$ is a Carathéodory function satisfying 4.10 and the following growth bound:

$$
|g(x, y, z)| \leq a(x, y)+b(x, y)|z|^{p} \text { for each } z \in \mathbb{R} \text { and a.e. }(x, y) \in \Omega,
$$

with $a \in L^{2}(\Omega ; W)$ and $b \in L^{2 /(1-p)}(\Omega ; W)$ if $p \in[0,1)$ and $b \in L^{\infty}(\Omega)$ if $p=1$. One has a well defined and continuous Nemytski operator

$$
\begin{array}{cl}
g_{\#}: L^{2}(\Omega ; W) & \rightarrow L^{2}(\Omega ; W) \\
u & \mapsto g(\cdot, u(\cdot))
\end{array}
$$

which maps bounded sets to bounded sets with the estimate

$$
\left\|g_{\#}(u)\right\|_{L^{2}(\Omega ; W)}^{2} \leq 2\left(\|a\|_{L^{2}(\Omega ; W)}^{2}+\|b\|_{L^{2 /(1-p)}(\Omega, W)}^{2}\|u\|_{L^{2}(\Omega ; W)}^{2 p}\right), \quad p \in[0,1] .
$$

These claims on $g_{\#}$ are standard (see e.g. Vainberg [26]) where it is enough to note that the weighted spaces $L^{p}(\Omega ; W)$ can be regarded as standard $L^{p}$ spaces with respect to the measure $\mu(E):=\int_{E} W d x d y$ which is absolutely continuous with respect to the Lebesgue measure. We will find weak solutions to (5.1) by solving a fixed point problem which characterizes the solutions in the following sense.

Lemma 5.1. Let $(\Omega, \sigma)$ be a Dirichlet pair for $L$ and let $W \in \mathcal{W}$. Let $g$ satisfy (4.10) and 5.2). Then the nonlinear map $\Phi: L^{2}(\Omega ; W) \rightarrow L^{2}(\Omega ; W)$ defined by

$$
\Phi=S \circ M_{W} \circ g_{\#}
$$


is compact and any fixed point $u \in L^{2}(\Omega ; W)$ of $\Phi$ belongs to $H_{0}^{1}(\Omega ; K)$ and is a weak solution of the Dirichlet problem (5.1). Conversely, any weak solution $u \in H_{0}^{1}(\Omega ; K) \subset L^{2}(\Omega ; W)$ of (5.1) is a fixed point of $\Phi$.

Proof. That $\Phi$ is well defined and compact follows from the considerations above. If $u \in L^{2}(\Omega ; W)$ is a fixed point of $\Phi$, then $u=S(h)$ for $h=M_{W} g_{\#} u \in L^{2}\left(\Omega ;|K|^{-1}\right)$ and hence $u \in H_{0}^{1}(\Omega ; K)$ and is a weak solution of 5.1.

Our first result uses the Leray-Schuader Principle for sublinear $g$.

Theorem 5.2. Let $\Omega, L$, and $W$ be as in Lemma 5.1. Let $g$ satisfy (4.10) and 5.2 with $p \in[0,1)$. Then there exists a weak solution $u \in H_{0}^{1}(\Omega ; K)$ to the Dirichlet problem (5.1).

Proof. The proof is identical to that of Theorem 4.2 of [14 for Tricomi type equations. We recall only the main points of the argument. Lemma 5.1 reduces the question to the existence of a fixed point for $\Phi$.

By the Leray-Schauder Principle, it is enough to show the following a priori bound: there exists a constant $C>0$ such that

$$
u=t \Phi(u), t \in(0,1) \Rightarrow\|u\|_{L^{2}(\Omega ; W)} \leq C .
$$

The case $p=0$ is obvious. Using $t \in(0,1)$, the boundedness of $T=S \circ M_{W}$ on $L^{2}(\Omega ; W)$ and the hypotheses on $a, b$ and $W$ one proves the existence of constants $C_{1}, C_{2}$ and $C_{3}$ such that

$$
\|u\|_{L^{2}(\Omega ; W)} \leq\|T\|_{\mathrm{op}}\left[C_{1}+C_{2}\|u\|_{L^{2}(\Omega ; W)}^{p}+C_{3}\|u\|_{L^{2}(\Omega ; W)}^{2 p}\right]^{1 / 2} .
$$

If (5.4) were to fail, then there would be a sequence $\left\{u_{n}\right\}_{n \in \mathbb{N}}$ satisfying $u_{n}=t_{n} \Phi\left(u_{n}\right)$ with $t_{n} \in(0,1)$ and $\left\|u_{n}\right\|_{L^{2}(\Omega ; W)} \rightarrow+\infty$. This contradicts 5.5 for $p \in(0,1)$.

Our next result uses the Contraction Mapping Principle for at most linear $g$ which can be applied to equations of the form (1.10), in which the derivatives $u_{x}$ and $u_{y}$ appear and where as usual $W$ is a weight function controlled by $|K|$, see (3.7). The hypotheses on $g=g(x, y, z, s, t)$ are the following three conditions. The function $g: \Omega \times \mathbb{R}^{3} \rightarrow \mathbb{R}$ satisfies the Carathéodory condition

$$
g \text { is measurable in }(x, y) \text { for each }(z, s, t) \in \mathbb{R}^{3} \text { and continuous in }(z, s, t) \text { for a.e. }(x, y) \in \Omega \text {. }
$$

One has the natural growth bound: for each $(z, s, t) \in \mathbb{R}^{3}$ and almost every $(x, y) \in \Omega$ one has

$$
|g(x, y, z, s, t)| \leq a(x, y)+b(x, y)|z|^{\beta}+c(x, y)|s|^{\gamma}+d(x, y)|t|^{\delta} .
$$

with given constants

$$
\beta, \gamma, \delta \in[0,1]
$$

and given non-negative functions

$$
a \in L^{2}(\Omega ; W), W^{\frac{1}{2}} b \in L^{\frac{2}{1-\beta}}(\Omega), W^{\frac{1-\gamma}{2}} c \in L^{\frac{2}{1-\gamma}}(\Omega), W^{\frac{1}{2}} d \in L^{\frac{2}{1-\delta}}(\Omega)
$$

with the convention that when $\beta=1, \gamma=1$ or $\delta=1$ then the corresponding function is essentially bounded on $\Omega$. Finally, one has the Lipschitz estimate in $(z, s, t)$ : for a.e. $(x, y) \in \Omega$

$$
\begin{aligned}
(W(x, y))^{\frac{1}{2}}\left|g\left(x, y, z_{1}, s_{1}, t_{1}\right)-g\left(x, y, z_{0}, s_{0}, t_{0}\right)\right| \leq & {\left[\alpha_{0}^{2}\left(z_{1}-z_{0}\right)^{2}\right.} \\
& \left.+\alpha_{1}^{2}\left(|K(x, y)|\left(s_{1}-s_{0}\right)^{2}+\left(t_{1}-t_{0}\right)^{2}\right)\right]^{1 / 2}
\end{aligned}
$$

with given constants $\alpha_{0}, \alpha_{1} \in \mathbb{R}$ satisfying

$$
\left(\alpha_{0}^{2} C_{P}^{2}+\alpha_{1}^{2}\right)^{1 / 2}<\min \left\{\left|\lambda_{1}^{-}\right|,\left|\lambda_{1}^{+}\right|\right\}
$$

where $\lambda_{1}^{ \pm}$are the positive/negative eigenvalues of minimum modulus of $L$ described in Theorem 3.6 and $C_{P}$ is the Poincaré constant in 2.4. 
Theorem 5.3. Let $(\Omega, \sigma)$ be a Dirichlet pair for $L$ and $g$ a nonlinearity satisfying (5.6), (5.7), (5.8), (5.9), (5.10) and 5.11). Then there exists a unique weak solution $u \in H_{0}^{1}(\Omega ; K)$ to the semilinear Dirichlet problem 1.10 .

Proof. The hypotheses (5.6), 5.7), (5.8) and (5.9) ensure that the Nemytskii operator

$$
\begin{array}{cl}
g_{\#}: H_{0}^{1}(\Omega ; K) & \rightarrow L^{2}(\Omega ; W) \\
u & \mapsto g\left(\cdot, u(\cdot), u_{x}(\cdot), u_{y}(\cdot)\right)
\end{array}
$$

is well defined and maps bounded sets to bounded sets with the estimate

$$
\begin{aligned}
\left\|g_{\#}(u)\right\|_{L^{2}(\Omega ; W)}^{2} & \leq 16\left[\|a\|_{L^{2}(\Omega ; W)}^{2}+C_{P}^{2 \beta}\left\|W^{\frac{1}{2}} b\right\|_{L^{\frac{2}{1-\beta}}(\Omega)}^{2}\|u\|_{H_{0}^{1}(\Omega ; K)}^{2 \beta}\right. \\
& \left.+\max \left\{C_{W}^{\gamma}\left\|W^{\frac{1-\gamma}{2}} c\right\|_{L^{\frac{2}{1-\gamma}(\Omega)}}^{2},\left\|W^{\frac{1}{2}} d\right\|_{L^{\frac{2}{1-\delta}(\Omega)}}^{2}\right\}\left(\left\|u_{x}\right\|_{L^{2}(\Omega,|K|)}^{2 \gamma}+\left\|u_{y}\right\|_{L^{2}(\Omega)}^{2 \delta}\right)\right],
\end{aligned}
$$

where $C_{P}$ is the Poincaré constant and where we recall that $0<W \leq C_{W}|K|$ a.e. in $\Omega$. Since $(\Omega, \sigma)$ is a Dirichlet pair for $L$, we have the continuous solution operator $S: L^{2}\left(\Omega ;|K|^{-1}\right) \rightarrow H_{0}^{1}(\Omega ; K)$ to the linear problem and hence weak solutions to 1.10 are precisely the fixed points of the nonlinear operator

$$
\Psi: H_{0}^{1}(\Omega ; K) \rightarrow H_{0}^{1}(\Omega ; K) \text { defined by } \Psi=S \circ M_{W} \circ g_{\#}=T \circ g_{\#} .
$$

The conditions (5.10) and 5.11) show that $\Psi$ is a contraction. Indeed, for $u, v \in H_{0}^{1}(\Omega ; K)$ one has

$$
\|\Psi(u)-\Psi(v)\|_{H_{0}^{1}(\Omega ; K)}^{2} \leq\|T\|_{\mathrm{op}}^{2}\left\|g_{\#}(u)-g_{\#}(v)\right\|_{L^{2}(\Omega ; W)}^{2}
$$

and using (5.10) one has

$$
\left\|g_{\#}(u)-g_{\#}(v)\right\|_{L^{2}(\Omega ; W)}^{2} \leq\left(\alpha_{0}^{2} C_{P}^{2}+\alpha_{1}^{2}\right)\|u-v\|_{H_{0}^{1}(\Omega ; K)}^{2} .
$$

Combining (5.14) and 5.15) one has

$$
\|\Psi(u)-\Psi(v)\|_{H_{0}^{1}(\Omega ; K)}^{2} \leq\|T\|_{\mathrm{op}}^{2}\left(\alpha_{0}^{2} C_{P}^{2}+\alpha_{1}^{2}\right)\|u-v\|_{H_{0}^{1}(\Omega ; K)}^{2} .
$$

Since the operator norm of $T$ depends on the spectral radius of $L$, the condition (5.11) yields

$$
\|T\|_{\text {op }}\left(\alpha_{0}^{2} C_{P}^{2}+\alpha_{1}^{2}\right)^{1 / 2}=\frac{1}{\min \left\{\left|\lambda_{1}^{-}\right|,\left|\lambda_{1}^{+}\right|\right\}}\left(\alpha_{0}^{2} C_{P}^{2}+\alpha_{1}^{2}\right)^{1 / 2}<1 .
$$

Hence $\Psi$ is a contraction.

Using this nonlinear perturbation result, we can address the question of solvability of the Dirichlet problem for linear equations with lower order terms; that is, for equations of the form

$$
L u=-W c_{0} u-W b_{1} u_{x}-W b_{2} u_{y}+W f
$$

where the coefficients $\left(c_{0}, b_{1}, b_{2}\right)$ and the source term $f$ are functions of $(x, y)$. Applying the above result with

$$
g(x, y, z, s, t)=-c_{0}(x, y) z-b_{1}(x, y) s-b_{2}(x, y) t+f(x, y)
$$

one sees that the growth conditions (5.7), (5.8) and (5.9) require $\beta, \gamma, \delta=1$ and hence one needs

$$
f \in L^{2}(\Omega ; W) \text { and } W^{\frac{1}{2}} c_{0}, b_{1}, W^{\frac{1}{2}} b_{2} \in L^{\infty}(\Omega) .
$$

Since $K$ vanishes on $\Omega \cap \Sigma$ and $0<W \leq C_{W}|K|$ a.e. on $\Omega$, the coefficients $\left(W c_{0}, W b_{1}, W b_{2}\right)$ must also vanish there as well; in particular, $W c_{0}, W b_{2}$ are dominated by $W^{\frac{1}{2}}$, while $W b_{1}$ is controlled by $W$. Finally, by the constraint (5.11), these coefficients must be small in a prescribed sense. We record the following corollary whose proof is now immediate. 
Corollary 5.4. Let $(\Omega, \sigma)$ be a Dirichlet pair for $L=-\operatorname{div}\left(K D_{x}, D_{y}\right)$ and let $W$ be a weight controlled by $|K|$. For every $f \in L^{2}(\Omega ; W)$ there exists a unique weak solution $u \in H_{0}^{1}(\Omega ; K)$ to the Dirichlet problem

$$
\begin{cases}-\left(K u_{x}\right)_{x}-u_{y y}+W b_{1} u_{x}+W b_{2} u_{y}+W c_{0} u=W f & \text { in } \Omega \\ u=0 & \text { on } \partial \Omega\end{cases}
$$

provided that

$$
\max \left\{C_{W}\left\|b_{1}\right\|_{L^{\infty}(\Omega)}^{2},\left\|W^{\frac{1}{2}} b_{2}\right\|_{L^{\infty}(\Omega)}^{2}\right\}+C_{P}^{2}\left\|W^{\frac{1}{2}} c_{0}\right\|_{L^{\infty}(\Omega)}^{2}<\frac{1}{3}\left[\min \left\{\left|\lambda_{1}^{-}\right|,\left|\lambda_{1}^{+}\right|\right\}\right]^{2},
$$

where $\lambda_{1}^{ \pm}$are the positive/negative eigenvalues of minimum modulus of $L$ described in Theorem 3.6 and $C_{P}$ is the Poincaré constant in 2.4.

We conclude this section with some remarks about the fixed point methods used here.

Remark 5.5. The hypothesis (5.10) - 5.11) used in the nonlinear results (and in the coefficient bounds 5.17) for the linear problem 5.16 with lower order terms) are stated in terms of the spectral radius $\lambda_{1}:=\min \left\{\left|\lambda_{1}^{-}\right|,\left|\lambda_{1}^{+}\right|\right\}$and the Poincaré constant $C_{P}$. These values are unknown, but may be estimated. In particular, since one can prove the Poincaré inequality (2.4) by integrating along segments in the $y$-direction, the Poincaré constant can be estimated in terms of the diameter $\operatorname{diam}_{y}(\Omega)$ of $\Omega$ in the $y$-direction and $C_{P}$ is an increasing function of $\operatorname{diam}_{y}(\Omega)$. In addition, the constant $C$ in the admissibility estimate $(3.3)$ gives an upper bound on the norm of the solution operator $S: L^{2}\left(\Omega ;|K|^{-1}\right) \rightarrow H_{0}^{1}(\Omega ; K)$. The reciprocal of this upper bound gives a lower bound on the sharp value of $\lambda_{1}$.

\section{Proof of Theorem 3.3}

The technique is an integral variant of the classical $(a, b, c)$-multiplier method of Friedrichs [11] and Morawetz [17. This integral variant was first presented by Didenko [7] for Tricomi type equations with the homogeneous Dirichlet condition placed on a proper subset of the boundary. The method was extended to Tricomi type equations with the Dirichlet condition imposed everywhere in [12] and then adapted to the PDE in (1.1) by Otway 21] (see [23] for a survey and comparison of multiplier methods for mixed type equations). We will follow here the scheme as presented in Appendix A of [13], which covers a small technical gap in the original proof [12], which was repeated in [21]. See Remark 6.4 after the proof.

Proof of Theorem 3.3. The main idea in the proof is to obtain suitable apriori estimates by estimating from above and below the integral expression

$$
\mathcal{I}=\int_{\Omega} \psi L \varphi d x d y=\int_{\Omega} \psi L M \psi d x d y
$$

for $\varphi \in C_{0}^{\infty}(\Omega)$ and $\psi$ a multiplier constructed as the solution to the auxiliary singular Cauchy problem

$$
\left\{\begin{array}{l}
M \psi:=a \psi+b \psi_{x}+c \psi_{y}=\varphi \text { in } \Omega \\
\psi=0 \text { on } \partial \Omega \backslash O
\end{array}\right.
$$

where the coefficients $(a, b, c)$ are to be selected so that $\psi$ lives in the desired solution space and that the integral in 6.1 yields a positive definite quadratic form in $\psi$. The problem is singular in the sense that the vector field $V=(b, c)$ will have a sink in $O \in \partial \Omega$ and the flow of $V$ transports $\partial \Omega \backslash O$ into $O$. A suitable choice of multipliers $\psi$ results from selecting

$$
(b, c)=(-\beta x,-y) \text { for some } \beta>m+1
$$

and

$$
a=a_{0}+a_{1} x^{\tau}:=\frac{\beta-m-1}{2}-\frac{1}{4 X^{\tau}} x^{\tau} \quad \text { where } X:=\sup _{(x, y) \in \Omega} x \text { and } \tau \in(0,1) .
$$


Notice that $V=(b, c)$ is the vector field used in the star-shaped hypothesis 3.5 . We also recall that $m \geq 1$ is the parameter appearing in the hypotheses (1.6) and 1.7 concerning the function $\sigma$ in the expression $K(x, y)=x-\sigma(y)$.

Associated to the parameter $\tau \in(0,1)$, we make use of the space $H_{0}^{1}(\Omega ; K, \tau)$ defined as the completion of $C_{0}^{1}(\Omega)$ with respect to the norm

$$
\|\psi\|_{H_{0}^{1}(\Omega ; K, \tau)}=\left[\int_{\Omega}\left(|x|^{\tau}|K| \psi_{x}^{2}+\psi_{y}^{2}\right) d x d y\right]^{1 / 2} .
$$

Claim 1: For each $\varphi \in C_{0}^{\infty}(\Omega)$ and each $\tau \in(0,1)$ there exists a solution $\psi \in C^{\infty}(\Omega) \cap C^{0}(\bar{\Omega})$ to the problem 6.2. Moreover, $\psi \in C^{\infty}\left(\bar{\Omega} \backslash B_{r}(O)\right)$ for each small $r>0$ and $\|\psi\|_{H_{0}^{1}(\Omega ; K, \tau)}<+\infty$ for each $\tau \in(0,1)$.

Indeed, the hypothesis 3.5 ensures that all complete characteristic curves $\gamma$ for the Cauchy problem 6.2. which start from an endpoint on $\partial \Omega \backslash O$ will remain in $\Omega$ until reaching their terminal point in $O$. By the hypotheses (3.4), the coefficient $a$ is also smooth on $\bar{\Omega} \backslash O$ and hence the unique solution $\psi$ will be $C^{\infty}\left(\bar{\Omega} \backslash B_{r}(O)\right)$ for each small $r$. The compact support of $\varphi$ implies that $\psi$ vanishes in a neighborhood of each point $z \in \partial \Omega \backslash O$. To complete the regularity claims, one uses the method of characteristics to obtain an explicit representation formula for $\psi$ where the ODE to be solved along each $\gamma$ is linear and the characteristics have the form $y=y_{0}\left(x / x_{0}\right)^{1 / \beta}$ with $\left(x_{0}, y_{0}\right) \in \partial \Omega \backslash\{O\}$. The compact support of $\varphi$ yields two critical values $\left(x_{0}^{ \pm}, y_{0}^{ \pm}\right)$for which the associated characteristics

$$
\gamma_{0}^{ \pm}=\left\{\left(x, \xi_{0}^{ \pm} x^{1 / \beta}\right): 0<x<x_{0}^{ \pm}, \quad \xi_{0}^{ \pm}=y_{0}^{ \pm} /\left(x_{0}^{ \pm}\right)^{1 / \beta}\right\}
$$

are tangent to the support of $\varphi$ and all characteristics starting from boundary points closer to $O$ along the boundary will remain outside of the support of $\varphi$. Moreover there exists $x_{1}>0$ so that $\varphi(x, y)$ vanishes for each $(x, y) \in \Omega$ with $x>x_{1}$. By choosing $x_{1}>0$ smaller if necessary, we may suppose that 1.6 holds for every $y$ with $(x, y) \in \overline{\mathcal{R}}_{x_{1}}$. It suffices to represent $\psi$ in the region

$$
\mathcal{R}_{x_{1}}=\left\{(x, y) \in \Omega: \quad \xi_{0}^{-} x^{1 / \beta}<y<\xi_{0}^{+} x^{1 / \beta}, \quad 0<x<x_{1}\right\} .
$$

With $(b, c)=(-\beta x,-y)$ and $a=a_{0}+a_{1} x^{\tau}$, the solution $\psi$ in $\mathcal{R}_{x_{1}}$ can be represented as

$$
\psi(x, y)=C_{1} h\left(\left(\frac{x}{x_{1}}\right)^{-\frac{1}{\beta}} y\right) x^{\frac{a_{0}}{\beta}} e^{\frac{a_{1} x^{\tau}}{\beta \tau}} \text { with } C_{1}=x_{1}^{-\frac{a_{0}}{\beta}} e^{-\frac{a_{1} x_{1}^{\tau}}{\beta \tau}},
$$

where $h(y)=\psi\left(x_{1}, y\right)$ for $y \in\left[y_{1}^{-}, y_{1}^{+}\right]=\left[\xi_{0}^{-} x_{1}^{1 / \beta}, \xi_{0}^{+} x_{1}^{1 / \beta}\right]$ gives the new initial data on the vertical boundary segment of $\mathcal{R}_{x_{1}}$. It follows that $\psi(x, y) \rightarrow 0$ as $(x, y) \rightarrow O$ so that $\psi \in C^{0}(\bar{\Omega})$ and has zero trace on the boundary. Using $(6.8)$ one obtains that the norm 6.5$)$ is finite provided that

$$
a_{0}>0, a_{1}<0, \tau>0 \text { and } \frac{2 a_{0}+m+1}{\beta}+\tau>1 .
$$

The choices (6.3) and (6.4) ensure the validity of 6.9).

Claim 2: There exists $C=C(\Omega, \sigma)$ such that for each $\tau \in(0,1)$ one has

$$
\|\varphi\|_{L^{2}(\Omega ;|K|)} \leq C|| L \varphi \|_{H^{-1}(\Omega ; K, \tau)}, \quad \varphi \in C_{0}^{\infty}(\Omega)
$$

where $\|\cdot\|_{H^{-1}(\Omega ; K, \tau)}$ is the norm on the dual space to $H_{0}^{1}(\Omega ; K, \tau)$.

We begin by estimating the integral 6.1 from below. Since the integrand has compact support, for each small $h>0$ one has

$$
\mathcal{I}=\mathcal{I}_{h}:=\int_{\Omega_{h}} \psi L \varphi d x d y
$$


where $\Omega_{h}:=\Omega \cap\{(x, y) \in \Omega: x>h\}$. The integrand has regularity limited only by the coefficient $K$ (which is $C^{1}$ ) and so one can integrate by parts to find

$$
\mathcal{I}=\mathcal{I}_{h}=\frac{1}{2} \int_{\Omega_{h}}\left(\alpha \psi_{x}^{2}+\gamma \psi_{y}^{2}+\delta \psi^{2}\right) d x d y-\frac{1}{2} \int_{\Gamma_{h}}\left[\left(K \psi_{x}^{2}+\psi_{y}^{2}\right) b+K a_{x} \psi^{2}\right] d y:=\mathcal{A}_{h}+\mathcal{B}_{h}
$$

where $\Gamma_{h}:=\{(h, y) \in \Omega\}$ and

$$
\begin{gathered}
\alpha=K\left(2 a+b_{x}-c_{y}\right)-b K_{x}-c K_{y}=K\left(2 a_{0}+2 a_{1} x^{\tau}-\beta+1\right)+\beta x-y \sigma^{\prime}(y) \\
\gamma=2 a-b_{x}+c_{y}=2 a_{0}+2 a_{1} x^{\tau}+\beta-1 \\
\delta=L a=-a_{1} \tau x^{\tau-2}[\tau x+(1-\tau) \sigma(y)]
\end{gathered}
$$

We first find a lower bound on the area integral $\mathcal{A}_{h}$. Using $\tau \in[0,1], a_{1}<0, x>0$ and $\sigma(y) \geq 0$ in (6.14), one has

$$
\delta \geq 0 \text { on } \Omega \text {. }
$$

Making the choices of $a_{0}$ and $a_{1}$ in (6.4), $\beta>m+1$ in (6.3) and $m \geq 1$, one estimates (6.13) by

$$
\gamma>m+2 a_{1} X^{\tau}=m-1 / 2 \geq 1 / 2 \text { on } \Omega .
$$

On $\Omega^{+}$, where $K=x-\sigma(y)>0$, one estimates 6.12 by

$$
\alpha=|K|\left(\beta-m+2 a_{1} x^{\tau}\right)+\beta \sigma(y)-y \sigma^{\prime}(y)>\frac{1}{2 X^{\tau}}|x|^{\tau}|K| \text { on } \Omega^{+},
$$

where we have used also the condition 1.7$)$ and $\beta>m$. Similarly, on $\Omega^{-}$, where $K=x-\sigma(y)<0$, one has

$$
\alpha=|K|\left(-2 a_{1} x^{\tau}\right)+m \sigma(y)-y \sigma^{\prime}(y)+(\beta-m) x>\frac{1}{2 X^{\tau}}|x|^{\tau}|K| \text { on } \Omega^{-} .
$$

Combining 6.15 - 6.18), one has the lower bound

$$
\mathcal{A}_{h} \geq \frac{1}{4} \min \left\{1, \frac{1}{X^{\tau}}\right\} \int_{\Omega_{h}}\left(|x|^{\tau}|K| \psi_{x}^{2}+\psi_{y}^{2}\right) d x d y:=C_{\tau} \int_{\Omega_{h}}\left(|x|^{\tau}|K| \psi_{x}^{2}+\psi_{y}^{2}\right) d x d y .
$$

Next, we will show how to control the boundary integral $\mathcal{B}_{h}$ in the limit as $h \rightarrow 0^{+}$. Inserting the expressions for $K, a$ and $b$ one has

$$
\begin{aligned}
\mathcal{B}_{h} & =\frac{1}{2} \int_{\Gamma_{h}}\left[\left((h-\sigma(y)) \psi_{x}^{2}+\psi_{y}^{2}\right) \beta h-(h-\sigma(y)) \tau a_{1} h^{\tau-1} \psi^{2}\right] d y \\
& \geq \frac{\beta h}{2} \int_{\Gamma_{h}}\left((h-\sigma(y)) \psi_{x}^{2} d y+\frac{\tau h^{\tau-1}}{8 X^{\tau}} \int_{\Gamma_{h}}(h-\sigma(y)) \psi^{2} d y:=\mathcal{B}_{1}(h)+\mathcal{B}_{2}(h) .\right.
\end{aligned}
$$

For each $h<x_{1}$, one can use the representation formula 6.8 for $\psi$ whose support lines in the region (6.7). The integrals in 6.20 reduce to integrals over $\widetilde{\Gamma}_{h}:=\left\{(h, y): \xi_{0}^{-} h^{1 / \beta}<y<\xi_{0}^{+} h^{1 / \beta}\right\}$ determined by the characteristics (6.6). For $h$ sufficiently small one can apply the estimate 1.6$)$ to give $|h-\sigma(y)| \leq h+C_{0}|y|^{m}$ with $m \geq 1$ and one easily obtains

$$
\lim _{h \rightarrow 0^{+}}\left|\mathcal{B}_{2}(h)\right|=0 .
$$

To control the remaining boundary term $\mathcal{B}_{1}(h)$, one again exploits 6.8 and 1.6 to show that

$$
\mathcal{B}_{1}(h)+\frac{1}{2} \mathcal{A}_{h} \geq 0 \text { for each small } h>0,
$$

where it is enough to have (6.22) along a sequence $\left\{h_{n}\right\}_{n \in \mathbb{N}}$ with $h_{n} \rightarrow 0^{+}$. Indeed, for each $h$ small one can estimate 6.22 from below by

$$
\frac{\beta}{2} \int_{\widetilde{\Gamma}_{h}} h(h-\sigma(y)) \psi_{x}^{2} d y+C_{\tau} \int_{\widetilde{\Omega}_{h}} x^{\tau}(\sigma(y)-h) \psi_{x}^{2} d x d y,
$$


where $\widetilde{\Omega}_{h}:=\left\{(x, y) \in \Omega_{h} \cap \mathcal{R}_{x_{1}}:|y|^{m}>x / C_{0}\right\}$ and $\mathcal{R}_{x_{1}}$ is the region defined as in 6.7). Using (6.8) and Fubini's theorem one finds 6.22 .

Combining 6.11), 6.19, 6 (6.20), 6.21) and 6.22) one finds

$$
\mathcal{I}=\mathcal{I}_{h} \geq \frac{1}{8} \min \left\{1, \frac{1}{X^{\tau}}\right\} \int_{\Omega_{h}}\left(|x|^{\tau}|K| \psi_{x}^{2}+\psi_{y}^{2}\right) d x d y+o(h), \text { as } h \rightarrow 0^{+},
$$

and passing to the limit yields the lower bound

$$
\mathcal{I} \geq \frac{1}{8} \min \left\{1, \frac{1}{X^{\tau}}\right\}\|\psi\|_{H_{0}^{1}(\Omega ; K, \tau)}^{2} .
$$

Estimating from above one has

$$
\mathcal{I} \leq\|\psi\|_{H_{0}^{1}(\Omega ; K, \tau)}\|L \varphi\|_{H^{-1}(\Omega ; K, \tau)},
$$

which when combined with 6.23 yields

$$
\|\psi\|_{H_{0}^{1}(\Omega ; K, \tau)} \leq 8 \max \left\{1, X^{\tau}\right\}\|L \varphi\|_{H^{-1}(\Omega ; K, \tau)} .
$$

Recalling that $\varphi=M \psi$, one estimates to find the existence of a constant $C_{M}=C_{M}(\Omega, \sigma)>0$ such that

$$
\|\varphi\|_{L^{2}(\Omega ;|K|)}=\|M \psi\|_{L^{2}(\Omega ;|K|)} \leq C_{M}\|\psi\|_{H_{0}^{1}(\Omega ; K, \tau)},
$$

which combined with 6.24 yields 6.10 with $C(\Omega, \sigma)=8 C_{M} \max \{1, X\}$ independent of $\tau$.

Claim 3: For each $f \in L^{2}\left(\Omega ;|K|^{-1}\right)$ there exists a unique weak solution $u \in H_{0}^{1}(\Omega ; K)$ of the Dirichlet problem 1.3 in the sense of Definition 3.1.

Using the estimate (6.10) and standard functional analysis, for each $\tau \in(0,1)$, there exists $u_{\tau} \in$ $H_{0}^{1}(\Omega ; K, \tau)$ such that

$$
\left\|u_{\tau}\right\|_{H_{0}^{1}(\Omega ; K, \tau)} \leq C(\Omega, \sigma)\|f\|_{L^{2}\left(\Omega ;|K|^{-1}\right)}
$$

and

$$
\int_{\Omega} u_{\tau} L \varphi d x d y=(f, \varphi)_{L^{2}(\Omega)}, \quad \varphi \in C_{0}^{\infty}(\Omega),
$$

where $C(\Omega, \sigma)$ is the constant of 6.10 .

One then considers the sequence $\left\{u_{1 / n}\right\}$ of these distributional solutions with $\tau_{n}=1 / n$. There is monotonicity in $\tau$ of the family of norms $(6.5)$; that is: for each pair $\tau, \tau^{\prime}$ with $0 \leq \tau<\tau^{\prime} \leq 1$ one has

$$
\|w\|_{H_{0}^{1}\left(\Omega ; K, \tau^{\prime}\right)} \leq(1+X)^{1 / 2}\|w\|_{H_{0}^{1}(\Omega ; K, \tau)}, \quad w \in H_{0}^{1}(\Omega ; K, \tau) .
$$

Using this monotonicity property, Lemma A.3 of [13] shows how to extract a subsequence which converges weakly in $L^{2}(\Omega)$ to a limit $u$ in $H_{0}^{1}(\Omega ; K)$ which satisfies the estimate

$$
\|u\|_{H_{0}^{1}(\Omega ; K)} \leq(1+X)^{1 / 2} C(\Omega, \sigma)\|f\|_{L^{2}\left(\Omega ;|K|^{-1}\right)},
$$

Moreover, $u$ is a distributional solution in the sense 6.25.

The distributional solution satisfying 6.26 can then be shown to be a weak solution in the sense of Definition 3.1. One merely repeats the argument of Lemma A.4 of [13] in which one considers a sequence $\left\{u_{n}\right\}_{n \in \mathbb{N}} \subset C_{0}^{\infty}(\Omega)$ which converges in the $H_{0}^{1}(\Omega ; K)$ norm to the distributional solution $u$. The uniqueness then follows as well by applying Lemma A.5 of [13].

Remark 6.4. In [21], which treated the case $\sigma(y)=y^{2}$, the additional term $\frac{1}{4 X^{\tau}} x^{\tau}$ in 6.4 is not present just as it was absent in the original proof [12] for the Tricomi type case. Without this additional term, the solution $\psi$ of 6.2 will lie in $C^{\infty}(\Omega) \cap C^{0}(\bar{\Omega})$, but it just fails to lie in $H_{0}^{1}(\Omega ; K)$, which corresponds to $\tau=0$. The proof of Lemma 2.1 of [21] (as well as the proof of Proposition 4.1 of [22]) repeats the erroneous claim made in Step 1 of the proof of Lemma 3.3 in [12. The correction in the Tricomi type case was made in 
Lemma A.1 of 13 in which the new term $\frac{1}{4 X^{\tau}} x^{\tau}$ improves the regularity of $\psi$ (while maintaining the needed positivity in 6.1 for the lower estimate) and the constant $\frac{\beta-m-1}{2}$ is calibrated in an optimal way.

\section{Proof of Lemmas 3.7 and 3.8}

In preparation for the proof of the compact embedding Lemma 3.7, we recall the following preliminaries taken from Danielli [6]. In all that follows $\Omega_{0}$ and $\Omega$ will be bounded open subsets of $\mathbb{R}^{N}$ such that $\bar{\Omega} \subset \Omega_{0}$ and $A: \Omega_{0} \rightarrow \operatorname{Sym}_{N}^{+}(\mathbb{R})$.

Definition 7.1. One says that $A$ defines a weak sub-Riemannian structure on $\Omega_{0}$ if for every $z_{0} \in \Omega_{0}$ there exist a neighborhood $W$ of $z_{0}$, a vector $\eta_{0} \in \mathbb{R}^{N}$, two positive numbers $\rho, t_{0}$ and a map

$$
\Phi:\left[0, t_{0}\right] \times W \times B_{\rho}\left(\eta_{0}\right) \rightarrow \Omega_{0}
$$

of class $C^{1}$ such that

$$
\begin{gathered}
t \mapsto \Phi(t, z, \eta)=x(t ; z, \eta) \text { is an } A \text {-subunit curve with } x(0 ; z, \eta)=z \text { for all } z \in W, \eta \in B_{\rho}\left(\eta_{0}\right) \\
\eta \mapsto \Phi(t, z, \eta) \text { is injective on } B_{\rho}\left(\eta_{0}\right) \\
\left|\operatorname{det}\left(\frac{\partial \Phi}{\partial \eta}(t, z, \eta)\right)\right|>0 \text { for all } t \in\left(0, t_{0}\right], z \in W, \eta \in B_{\rho}\left(\eta_{0}\right) .
\end{gathered}
$$

We also recall that $x:\left[0, t_{0}\right] \rightarrow \Omega_{0}$ is an $A$-subunit curve if it is absolutely continuous and satisfies

$$
\left|\left\langle x^{\prime}(t), \xi\right\rangle\right|^{2} \leq\langle A(x(t)) \xi, \xi\rangle \text { for a.e. } t \in\left(0, t_{0}\right) \text { and every } \xi \in \mathbb{R}^{n},
$$

where $\langle\cdot, \cdot\rangle$ is the standard inner product on $\mathbb{R}^{N}$. Finally, recalling that $W_{0}^{1, p}(\Omega ; A)$ is the completion of $C_{0}^{1}(\Omega)$ with respect to the norm (3.10), Proposition 3.1 of [6] states that: if $A: \Omega_{0} \rightarrow \operatorname{Sym}_{N}^{+}(\mathbb{R})$ defines a weak sub-Riemannian structure on $\Omega_{0}$, then the embedding of $W_{0}^{1, p}(\Omega ; A)$ into $L^{p}(\Omega)$ is compact.

Remark 7.2. Danielli uses the map $\Phi$ to define a suitable family of local displacements (indexed by the parameter $t$ ) which replace Euclidian translations in the standard mollifying procedure used to prove the compactness property in the classical case (where $A(x)$ is the identity matrix and the Sobolev space $W_{0}^{1, p}(\Omega ; A)$ reduces to $W_{0}^{1, p}(\Omega)$ ). Proposition 3.1 of [6] is then a corollary of a more general result (Theorem 2.8 of [6). The term weak in Definition 7.1 refers to condition (7.3) which weakens what was introduced by Franchi-Lanconelli [9] as a sub-Riemmanian structure. See also Remark 2.4 of Franchi [8].

Proof of Lemma 3.7. Given $A$ of the form $A(x)=\operatorname{diag}\left[\Lambda_{1}^{2}(x), \ldots, \Lambda_{N}^{2}(x)\right]$ with $\left\{\Lambda_{j}\right\}_{j=1}^{N}$ satisfying (3.12), (3.13) and (3.14), it suffices to show that for each $z_{0} \in \Omega_{0}$ the needed map $\Phi$ exists. Since $A$ is the square of $\operatorname{diag}\left[\Lambda_{1}, \ldots, \Lambda_{N}\right]$ with each $\Lambda_{j}$ non-negative and $C^{1}\left(\Omega_{0}\right)$, Remark 2.7 of [9] shows that $\Phi$ satisfying (7.1) can be constructed as the time $t$ flow of a suitable Cauchy problem. More precisely, fixing $z \in \Omega_{0}$ and $\eta \in \mathbb{R}^{N}$ with $|\eta| \leq 1$, an $A$-subunit curve $x=x(t ; z, \eta)$ satisfying $x(0 ; z, \eta)=z$ is given by the unique solution of

$$
\left\{\begin{array}{l}
x^{\prime}(t)=A^{1 / 2}(x(t)) \eta \\
x(0)=z
\end{array}\right.
$$

Indeed, as argued in [9], for each $\xi \in \mathbb{R}^{N}$ one has

$$
\left|\left\langle x^{\prime}(t), \xi\right\rangle\right|^{2}=\left|\left\langle\eta, A^{1 / 2}(x(t)) \xi\right\rangle\right|^{2} \leq\left|A^{1 / 2}(x(t)) \xi\right|^{2}|\eta|^{2} \leq\left|A^{1 / 2}(x(t)) \xi\right|^{2}=\langle A(x(t)) \xi, \xi\rangle .
$$

The system (7.4) is autonomous and $F$ defined by $F(x, \eta)=A^{1 / 2}(x) \eta$ belongs to $C^{1}\left(\Omega_{0} \times \mathbb{R}^{N}, \mathbb{R}^{N}\right)$. Hence one has $C^{1}$ dependence locally in $(t, z, \eta)$ and one defines the values of $\Phi$ by $\Phi(t, z, \eta)=x(t ; z, \eta)$, which by construction will take values in the bounded open set $\Omega_{0}$ and will be an $A$-subunit curve if one maintains 
$|\eta| \leq 1$. One needs only to show that a suitable neighborhood $\left[0, t_{0}\right] \times W \times B_{\rho}\left(\eta_{0}\right)$ exists so that 7.2$]$ and 7.3 hold.

For each $z_{0} \in \Omega_{0}$, we will select $\eta_{0}=1 /(2 \sqrt{N})(1, \ldots, 1)$ so that $\left|\eta_{0}\right|=1 / 2<1$ and pick $\rho<1 /(4 \sqrt{N})$ so that $B_{\rho}\left(\eta_{0}\right) \subset B_{1}(0)$. We will see below that this choice of $B_{\rho}\left(\eta_{0}\right)$ means that the $A$-subunit curves will have components which are increasing functions of $t$. We complete the definition of $\Phi$ by choosing a small pluri-rectangle $W$ containing $z_{0}$ and a small value $t_{0}$ for which the solutions of 7.4 starting from $W$ with parameter $\eta \in B_{\rho}\left(\eta_{0}\right)$ will remain in $\Omega_{0}$ for each $t \in\left[0, t_{0}\right]$. This can clearly be accomplished by a compactness argument and we will have $\Phi$ which satisfies 7.1 . For the properties 7.2 and 7.3 , the following claim plays a key role.

Claim 1: For each fixed $z \in \Omega_{0}$ and $\eta \in B_{\rho}\left(\eta_{0}\right)$, the unique solution of the system (7.4) satisfies

$$
x_{j}=x_{j}\left(t ; z_{1}, \ldots, z_{j}, \eta_{1}, \ldots, \eta_{j}\right)>z_{j} \text { for each } j=1 \ldots, N \text { and each } t \in\left(0, t_{0}\right] .
$$

The proof is by induction on $j$. Indeed, using 3.12 with $j=1$, one has

$$
x_{1}(t)=z_{1}+\eta_{1} \int_{0}^{t} \Lambda_{1}\left(x_{1}(s)\right) d s>z_{1} \text { for each } s \in\left(0, t_{0}\right]
$$

since $\eta_{1}>0$ and $\Lambda_{1}>0$ by (3.13). Assuming (7.5) for each index less than or equal to $j-1$, consider the index $j$. Using the structure condition (3.12, the positivity of $\eta_{j}$ and the non-negativity of $\Lambda_{j}$ one has

$$
x_{j}(t)=z_{j}+\eta_{j} \int_{0}^{t} \Lambda_{j}\left(x_{1}(s), \ldots, x_{j-1}(s), x_{j}(s)\right) d s \geq z_{j} \text { for each } s \in\left[0, t_{0}\right] .
$$

If there were $\bar{t} \in\left(0, t_{0}\right]$ such that $x_{j}(\bar{t})=z_{j}$, then by $(7.6)$ one would have

$$
\Lambda_{j}\left(x_{1}(s), \ldots, x_{j-1}(s), z_{j}\right)=0 \text { for every } s \in[0, \bar{t}] .
$$

For $s=0$ one has

$$
\Lambda_{j}\left(z_{1}, \ldots, z_{j-1}, z_{j}\right)=0
$$

and using the induction hypothesis one has $x_{k}(s)>z_{k}$ for each $s \in(0, \bar{t}]$ and for each $k=1, \ldots, j-1$. The components $x_{k}(s)$ are $C^{1}$ functions of $s \in[0, \bar{t}]$ and hence 7.7$)$ implies that there would exist $\varepsilon>0$ such that $S_{\varepsilon}\left(z_{1}, \ldots, z_{j-1}, z_{j}\right)$ as defined by 3.15 would contain an arc of a $C^{1}$ curve of positive length, which contradicts the condition (3.14). This completes the claim.

Claim 2: For each $\eta \in B_{\rho}\left(\eta_{0}\right)$ and for each $j \in\{1, \ldots, N\}$, one has

$$
\frac{\partial x_{j}}{\partial \eta_{j}}(t ; z, \eta)>0 \text { for each } t \in\left(0, t_{0}\right], z \in W .
$$

Indeed, again taking inspiration from Remark 2.7 of [9], we use the structure condition (3.12) to write

$$
x_{j}\left(t ; p_{j}\right)=z_{j}+\int_{0}^{t} \eta_{j} \Lambda_{j}\left(x_{1}\left(s ; p_{1}\right), \ldots, x_{j}\left(s ; p_{j}\right)\right) d s
$$

where we have denoted by $p_{j}:=\left(z_{1}, \ldots, z_{j}, \eta_{1}, \ldots, \eta_{j}\right)$. Hence $u_{j}:=\frac{\partial x_{j}}{\partial \eta_{j}}$ satisfies

$$
u_{j}\left(t ; p_{j}\right)=\int_{0}^{t}\left[\Lambda_{j}\left(x_{1}\left(s ; p_{1}\right), \ldots, x_{j}\left(s ; p_{j}\right)\right)+\eta_{j} \frac{\partial \Lambda_{j}}{\partial x_{j}}\left(s ; p_{j}\right) u_{j}\left(s ; p_{j}\right)\right] d s \quad \text { and } \quad u_{j}\left(0 ; p_{j}\right)=0,
$$

which is to say that $u_{j}\left(t, p_{j}\right)$ solves the Cauchy problem

$$
\left\{\begin{array}{l}
u_{j}^{\prime}\left(t ; p_{j}\right)=\Lambda_{j}\left(x_{1}\left(t ; p_{1}\right), \ldots, x_{j}\left(t ; p_{j}\right)\right)+\eta_{j} \frac{\partial \Lambda_{j}}{\partial x_{j}}\left(t ; p_{j}\right) u_{j}\left(t ; p_{j}\right) \\
u_{j}\left(0 ; p_{j}\right)=0
\end{array}\right.
$$

Hence

$$
u_{j}\left(t ; p_{j}\right)=\exp \left(\eta_{j} \int_{0}^{t} \frac{\partial \Lambda_{j}}{\partial x_{j}}\left(s ; p_{j}\right) d s\right) \int_{0}^{t} \exp \left(-\eta_{j} \int_{0}^{\tau} \frac{\partial \Lambda_{j}}{\partial x_{j}}\left(s ; p_{j}\right) d s\right) \Lambda_{j}\left(x_{1}\left(\tau ; p_{1}\right), \ldots, x_{j}\left(\tau ; p_{j}\right)\right) d \tau \geq 0 .
$$


If there were to exist a $\bar{t}$ such that $u_{j}(\bar{t})=0$, then $\Lambda_{j}\left(x_{1}\left(t ; p_{1}\right), \ldots, x_{j}\left(t ; p_{j}\right)\right)=0$ for every $t \in[0, \bar{t}]$. However, the relation (7.6) would then imply that $x_{j}\left(t ; z_{1}, \ldots, z_{j}, \eta_{1}, \ldots, \eta_{j}\right)=z_{j}$ for each $t \in[0, \bar{t}]$, which contradicts (7.5). This establishes Claim 2.

The remaining properties now follow easily. Since $x_{j}$ is independent of $\eta_{k}$ for $k>j$, the Jacobian determinant condition 7.3 is satisfied since

$$
\left|\operatorname{det}\left(\frac{\partial \Phi}{\partial \eta}(t ; z, \eta)\right)\right|=\left|\operatorname{det}\left(\frac{\partial x}{\partial \eta}(t ; z, \eta)\right)\right|=\left|\prod_{j=1}^{N} \frac{\partial x_{j}}{\partial \eta_{j}}(t ; z, \eta)\right|>0,
$$

where the positivity comes from 77.8. This condition also insures the injectivity condition (7.2). The argument is by contradiction. Suppose that for some $\left(z^{*}, t^{*}\right) \in W \times\left[0, t_{0}\right]$ one has

$$
x\left(t^{*} ; z^{*}, \eta\right)=x\left(t^{*} ; z^{*}, \tilde{\eta}\right) \text { for } \eta, \tilde{\eta} \in B_{\rho}\left(\eta_{0}\right) \text { with } \eta \neq \tilde{\eta} .
$$

Then for $j=1$, one has $x_{1}\left(t^{*} ; z_{1}^{*}, \eta_{1}\right)=x_{1}\left(t^{*} ; z_{1}^{*}, \tilde{\eta}_{1}\right)$ and hence there would be $\eta_{1}^{*}$ between $\eta_{1}$ and $\tilde{\eta}_{1}$ such that $\frac{\partial x_{1}}{\partial \eta_{1}}\left(t^{*} ; z_{1}^{*}, \eta_{1}^{*}\right)=0$, in contradiction with 7.8 . Hence $\eta_{1}=\tilde{\eta_{1}}$. for $j=2$, then one has $x_{2}\left(t^{*} ; z_{1}^{*}, z_{2}^{*}, \eta_{1}, \eta_{2}\right)=$ $x_{2}\left(t^{*} ; z_{1}^{*}, z_{2}^{*}, \eta_{1}, \tilde{\eta}_{2}\right)$. The same argument shows that $\eta_{2}=\tilde{\eta_{2}}$, and so on. Hence $\eta=\tilde{\eta}$, a contradiction.

Proof of Lemma 3.8. With $\Omega, Q, B_{r}\left(x_{0}\right), \zeta$ and $\delta$ as in the statement of the Lemma, we pick a cutoff function $\psi \in C_{0}^{\infty}(\mathbb{R})$ such that

$$
\psi \text { is even, } \operatorname{supp}(\psi) \subset\left[-\frac{1}{2}, \frac{1}{2}\right] \text { and } 0 \leq \psi \leq 1 .
$$

For $\alpha \geq 1$ and $B_{t}\left(x_{1}\right) \subset \subset B_{r}\left(x_{0}\right)$ we define the function $\varphi=\varphi_{t, x_{1}}(x)$ by

$$
\varphi(x):=\psi\left(\frac{\alpha^{2}-1}{t^{2}}\left|\left\langle x-x_{1}, \zeta\right\rangle\right|^{2}+\frac{1}{t^{2}}\left|x-x_{1}\right|^{2}\right):=\psi(s(x)) .
$$

Clearly $\varphi \in C_{0}^{\infty}(\Omega)$ and $\operatorname{supp}(\varphi) \subset \subset B_{t}\left(x_{1}\right) \subset \subset B_{r}\left(x_{0}\right)$. Direct calculation yields

$$
\begin{aligned}
\int_{\Omega}\langle D \varphi, Q D \varphi\rangle d x=\frac{4}{t^{4}} & \int_{\Omega}\left[\psi^{\prime}(s(x))\right]^{2}\left[\left(\alpha^{2}-1\right)^{2}\left\langle x-x_{1}, \zeta\right\rangle^{2}\langle Q(x) \zeta, \zeta\rangle\right. \\
& \left.+2\left(\alpha^{2}-1\right)\left\langle x-x_{1}, \zeta\right\rangle\left\langle Q(x) \zeta,\left(x-x_{1}\right)\right\rangle+\left\langle Q(x)\left(x-x_{1}\right), x-x_{1}\right\rangle\right] d x .
\end{aligned}
$$

In the case a), where $\langle Q(x) \zeta, \zeta\rangle \geq \delta>0$, for $x \in B_{r}\left(x_{0}\right)$ one finds

$$
\int_{\Omega}\langle D \varphi, Q D \varphi\rangle d x \geq \frac{4 \delta}{t^{4}} C_{1}\left(\alpha^{2}-1\right)^{2}-\frac{4 r^{2}}{t^{4}} C_{2}\left(2\left|\alpha^{2}-1\right|+1\right),
$$

with

$$
C_{1}=\int_{\Omega}\left[\psi^{\prime}(s(x))\right]^{2}\left|\left\langle x-x_{1}, \zeta\right\rangle\right|^{2} d x>0 \text { and } C_{2}=\int_{\Omega}\left[\psi^{\prime}(s(x))\right]^{2}\|Q(x)\|^{2}>0 .
$$

Selecting $\alpha \geq 1$ sufficiently large shows that the integral in 7.9 has a positive lower bound. In order to construct the desired collection $\left\{w_{1}, \ldots w_{n}\right\}$, one merely picks a finite sequence of mutually disjoint balls $B_{t_{1}}\left(x_{1}\right), \ldots, B_{t_{n}}\left(x_{n}\right)$ all contained in $B_{r}\left(x_{0}\right)$ and repeats the construction above to select $w_{i}=\varphi_{t_{i}, x_{i}}$.

Similarly, in the case b), where $\langle Q(x) \zeta, \zeta\rangle \leq-\delta<0$, for $x \in B_{r}\left(x_{0}\right)$ one finds

$$
\int_{\Omega}\langle D \varphi, Q D \varphi\rangle d x \leq-\frac{4 \delta}{t^{4}} C_{1}\left(\alpha^{2}-1\right)^{2}+\frac{4 r^{2}}{t^{4}} C_{2}\left(2\left|\alpha^{2}-1\right|+1\right),
$$

with $C_{1}$ and $C_{2}$ given again by 7.10 . Again selecting $\alpha \geq 1$ large shows that the integral in 7.11 has a negative upper bound. The desired collection $\left\{w_{1}, \ldots w_{n}\right\}$ is then constructed as before. 


\section{REFERENCES}

[1] Čanić, S. and Keyflitz, B.L. (1996). A smooth solution for a Keldysh type equation. Comm. Partial Differential Equations 21, 319-340.

[2] Čanić, S. and Keyflitz, B.L. (1996). An elliptic problem arising from the unsteady transonic small disturbance equation. J. Differential Equations 125, 548-574.

[3] Čanić, S. and Keyflitz, B.L. (1998). Riemann problems for the two-dimensional unsteady transonic small disturbance equation. SIAM J. Appl. Math. 58, 636-665.

[4] Čanić, S. and Keyflitz, B.L. (1998). Quasi-one-dimensional Riemann problems and their role in selfsimilar two-dimensional problems. Arch. Rational Mech. Anal. 144, 233-258.

[5] Chen, S.X. (2012). A mixed equation of Tricomi-Keldysh type. J. Hyperbolic Differ. Equ. 9, 545-553.

[6] Danielli, D. (1991). A compact embedding theorem for a class of degenerate Sobolev spaces. Rend. Sem. Mat. Univ. Polit. Torino 49, 399-420.

[7] Didenko, V.P. (1973). On the generalized solvability of the Tricomi problem. Ukrain. Math. J. 25, 10-18.

[8] Franchi, B. (1984). Propriétés des courbes intégrales de champs de vecteurs et estimations ponctuelles d'équations elliptiques dégénérées Goulaouic-Meyer-Schwartz Seminar, 1983 - 1984 Exp. No. 3, 14 pp.

[9] Franchi, B. and Lanconelli, E. (1985). Une condition géométrique pour l'enǵalité de Harnack J. Math. Pures Appl (9) 65, 237-256.

[10] Frankl', F.I. (1945). On the problems of Chaplygin for mixed sub- and supersonic flows Isv. Akad. Nauk. USSR Ser. Mat. 9, 121-143.

[11] Friedrichs, K.O. (1958). Symmetric positive linear differential equations. Comm. Pure Appl. Math. 11, 333-418.

[12] Lupo, D., Morawetz, C.S. and Payne, K.R. (2007). On closed boundary value problems for equations of mixed elliptic-hyperbolic type. Comm. Pure Appl. Math. 60(5), 1319-1348.

[13] Lupo, D., Monticelli, D.D. and Payne, K.R (2012). Spectral theory for linear operators of mixed type and applications to nonlinear Dirichlet problems, Comm. Partial Differential Equations 37 (9), 1495-1516.

[14] Lupo, D., Monticelli, D.D. and Payne, K.R. (2013). Fredholm properties and nonlinear Dirichlet problems for mixed type operators. J. Math. Anal. Appl. 397(2), 837-860.

[15] Lupo, D., Monticelli, D.D. and Payne, K.R. (2013). Variational characterizations of weak solutions to the Dirichlet problem for mixed type equations. Comm. Pure Appl. Math., to appear (DOI: 10.1002/cpa.21529).

[16] Monticelli, D.D. and Payne, K.R. (2009). Maximum principles for weak solutions of degenerate elliptic equations with a uniformly elliptic direction. J. Differential Equations 247, 1993-2026.

[17] Morawetz, C.S. (1958). A weak solution for a system of equations of mixed elliptic-hyperbolic type. Comm. Pure Appl. Math. 11, 315-331.

[18] Morawetz, C.S. (1970). The Dirichlet problem for the Tricomi equation. Comm. Pure Appl. Math. 23, $587-601$.

[19] Morawetz, C.S. (2004). Mixed equations and transonic flow. J. Hyperbolic Differ. Equ. 1, 1-26.

[20] Morawetz, C.S, Stevens, D.C. and Weitzner, H. (1985). A numerical experiment on a second-order partial differential equation of mixed type. Comm. Pure Appl. Math. 44, 1091-1106.

[21] Otway, T.H. (2010). Unique solutions to boundary value problems in the cold plasma model. SIAM. J. Math. Anal. 42, 3045-3053.

[22] Otway, T.H. (2012). The Dirichlet problem for elliptic-hyperbolic equations of Keldysh type, Volume 2043 of Lecture Notes in Mathematics. New York: Springer

[23] Payne, K.R. (2007). Multiplier methods for mixed type equations. Int. J. Appl. Math. Stat. 8, 58-75.

[24] Piliya, A.D. and Fedorov, V.I. (1971). Singularities of the field of an electromagnetic wave in a cold anisotropic plasma with two-dimensional inhomogeneity. Sov. Phys. JETP 33, 210-215. 
[25] Rabinowitz, P.H. (1986). Minimax Methods in Critical Point Theory with Applications to Differential Equations, Volume 65 of CBMS Regional Confererence Series in Mathematics. Providence, RI: American Mathematical Society.

[26] Vainberg, M.M. (1964). Variational Methods for the Study of Nonlinear Operators, San Francisco: Holden-Day Inc.

[27] Weitzner, H. (1984). Wave propagation based on the cold plasma model. Courant Institute MagentoFluid Dynamics Division Report. MF-103, August 1984, 49pp.

[28] Weitzner, H. (1985). Lower hybrid waves in the cold plasma model. Comm. Pure Appl. Math. 38, 919-932.

[29] Weitzner, H. (2012). Studies in the dynamics of a plasma. Comm. Pure Appl. Math. 65, 975-991.

Dipartimento di Matematica "F. Brioschi", Politecnico di Milano, P.zza L. Da Vinci, 32, 20133-Milano, Italy

E-mail address: daniela.lupo@polimi.it (Daniela Lupo)

Dipartimento di Matematica "F. Enriques", Università di Milano, Via C. Saldini 50, 20133-Milano, Italy

E-mail address: dario.monticelli@unimi.it (Dario D. Monticelli)

Dipartimento di Matematica "F. Enriques", Università di Milano, Via C. Saldini 50, 20133-Milano, Italy

E-mail address: kevin.payne@unimi.it (Kevin R. Payne) 